\title{
MULTIPLE-PRIORS OPTIMAL INVESTMENT IN DISCRETE TIME FOR UNBOUNDED UTILITY FUNCTION
}

\author{
BY ROMAIN BLANCHARD* AND LAURENCE CARASSUS ${ }^{\dagger}, *, 1$ \\ Université Reims Champagne-Ardenne* and \\ Léonard de Vinci Pôle Universitaire Research Center ${ }^{\dagger}$
}

This paper investigates the problem of maximizing expected terminal utility in a discrete-time financial market model with a finite horizon under nondominated model uncertainty. We use a dynamic programming framework together with measurable selection arguments to prove that under mild integrability conditions, an optimal portfolio exists for an unbounded utility function defined on the half-real line.

1. Introduction. We consider investors trading in a multi-period and discretetime financial market. We study the problem of terminal wealth expected utility maximisation under Knightian uncertainty. It was first introduced by F. Knight [Knight (1921)] and refers to the "unknown unknown," or uncertainty, as opposed to the "known unknown," or risk. This concept is very appropriate in the context of financial mathematics as it describes accurately market behaviors which are becoming more and more surprising. The belief of investors are modeled with a set of probability measures rather than a single one. This can be related to model misspecification issues or model risk and has triggered a renewed and strong interest by practitioners and academics alike.

The axiomatic theory of the classical expected utility was initiated by von Neumann and Morgenstern (1947). They provided conditions on investor preferences under which the expected utility of a contingent claim $X$ can be expressed as $E_{P} U(X)$ where $P$ is a given probability measure and $U$ is a so-called utility function. The problem of maximising the von Neumann and Morgenstern expected utility has been extensively studied; we refer to Rásonyi and Stettner (2005) and Rásonyi and Stettner (2006) for the discrete-time case and to Kramkov and Schachermayer (1999) and Schachermayer (2001) for the continuous-time one. In the presence of Knightian uncertainty, Gilboa and Schmeidler (1989) provided a pioneering contribution by extending the axiomatic of von Neumann and Morgenstern. In this case, under suitable conditions on the investor preferences, the

Received October 2016; revised May 2017.

${ }^{1}$ Supported by LPMA (UMR 7599).

MSC2010 subject classifications. Primary 93E20, 91B70, 91B16; secondary 91G10, 28B20, 49L20.

Key words and phrases. Knightian uncertainty, multiple-priors, optimal investment, nondominated model. 
utility functional is of the form $\inf _{P \in \mathcal{Q}^{T}} E_{P} U(X)$ where $\mathcal{Q}^{T}$ is the set of all possible probability measures representing the agent beliefs. Most of the literature on the so-called multiple-priors or robust expected utility maximisation assumes that $\mathcal{Q}^{T}$ is dominated by a reference measure. We refer to Schied, Föllmer and Weber (2009) for an extensive survey.

However, assuming the existence of a dominating reference measure does not always provide the required degree of generality from an economic and practical perspective. Indeed, uncertain volatility models [see Avellaneda, Levy and Paras (1996), Denis and Martini (2006), Lyons (1995)] are concrete examples where this hypothesis fails. On the other hand, assuming a nondominated set of probability measures significantly raises the mathematical difficulty of the problem as some of the usual tools of probability theory do not apply. In the multiple-priors nondominated case, Denis and Kervarec (2013) obtained the existence of an optimal strategy, a worst case measure as well as some "minmax" results under some compacity assumption on the set of probability measures and with a bounded (from above and below) utility function. This result is obtained in the continuous-time case. In the discrete-time case, Nutz (2016) (where further references to multiplepriors nondominated problematic can be found) obtained the first existence result without any compacity assumption on the set of probability measures but for a bounded (from above) utility function. We also mention two articles subsequent to our contribution. The first one [see Bartl (2016)] provides a dual representation in the case of an exponential utility function with a random endowment, and the second one [see Neufeld and Sikic (2016)] study a market with frictions in the spirit of Pennanen and Perkkio (2012) for a bounded from above utility function.

To the best of our knowledge, this paper provides the first general result for unbounded utility functions assuming a nondominated set of probability measures (and without compacity assumption). This includes, for example, the useful case of Constant Relative Risk Aversion utility functions (i.e., logarithm or power functions). In Theorem 1.11, we give sufficient conditions for the existence of an optimizer to our "maxmin" problem (see Definition 1.9). We work under the framework of Bouchard and Nutz (2015) and Nutz (2016). The market is governed by a nondominated set of probability measures $\mathcal{Q}^{T}$ that determines which events are relevant or not. Assumption 1.1, which is related to measurability issues, is the only assumption made on $\mathcal{Q}^{T}$ and is the cornerstone of the proof. We introduce two integrability assumptions. The first one (Assumption 3.1) is related to measurability and continuity issues. The second one (Assumption 3.5) replaces the boundedness assumption of Nutz (2016) and allows us to use auxiliary functions which play the role of properly integrable bounds for the value functions at each step. The noarbitrage condition is essential as well; we use the one introduced in Bouchard and Nutz (2015) and propose a "quantitative" characterisation in the spirit of Jacod and Shiryaev (1998) and Rásonyi and Stettner (2005). Finally, we introduce an alternative "strong" no-arbitrage condition (the $s N A$, see Definition 2.4) and prove in 
Theorem 3.6 that under the $s N A$ condition, Theorem 1.11 applies to a large range of settings.

As in Bouchard and Nutz (2015) and Nutz (2016) our proof relies heavily on measure theory tools, namely on analytic sets. Those sets display the nice property of being stable by projection or countable unions and intersections; however, they fail to be stable by complementation, hence the sigma-algebra generated by analytic sets contains sets that are not analytic which leads to significant measurability issues. Such difficulties arise for instance in Lemma 3.26, where we are still able to prove some tricky measurability properties, as well as in Proposition 3.30 which is pivotal in solving the dynamic programming. Note as well that we have identified (and corrected) a small issue in Lemma 4.12 of Bouchard and Nutz (2015), which is also used in Nutz (2016) to prove some important measurability properties. Indeed it is not enough in order to have joint-measurability of a function $\theta(\omega, x)$ to assume that $\theta(\cdot, x)$ is measurable and $\theta(\omega, \cdot)$ is lower-semicontinuous, one has to assume for example that $\theta(\omega, \cdot)$ is convex [see Lemma A.35 as well as the counter-example A.34].

To solve our optimisation problem, we follow a similar approach as Nutz (2016). We first consider a one-period case with strategy in $\mathbb{R}^{d}$. To "glue" together the solutions found in the one-period case, we use dynamic programming as in Rásonyi and Stettner (2005, 2006), Carassus and Rásonyi (2016), Carassus, Rásonyi and Rodrigues (2015), Nutz (2016) and Blanchard, Carassus and Rásonyi (2016) together with measurable selection arguments (Auman and Jankov-von Neumann theorems).

In the remainder of the Introduction, we recall some important properties of analytic sets, present our framework and state our main result. In Section 2, we prove our quantitative version of the multiple-priors no-arbitrage condition. In Section 3, we solve the expected utility maximisation problem, first in the one period case. Finally, the Appendix collects some technical results and proofs as well as some counter-examples to Bouchard and Nutz (2015), Lemma 4.12.

1.1. Polar sets and universal sigma-algebra. For any Polish space $X$ (i.e., complete and separable metric space), we denote by $\mathcal{B}(X)$ its Borel sigma-algebra and by $\mathfrak{P}(X)$ the set of all probability measures on $(X, \mathcal{B}(X))$. We recall that $\mathfrak{P}(X)$ endowed with the weak topology is a Polish space [see Bertsekas and Shreve (2004), Proposition 7.20, page 127, Proposition 7.23, page 131]. If $P$ in $\mathfrak{P}(X)$, $\mathcal{B}_{P}(X)$ will be the completion of $\mathcal{B}(X)$ with respect to $P$ and the universal sigmaalgebra is defined by $\mathcal{B}_{c}(X):=\bigcap_{P \in \mathfrak{P}(X)} \mathcal{B}_{P}(X)$. It is clear that $\mathcal{B}(X) \subset \mathcal{B}_{c}(X)$. In the rest of the paper, we will use the same notation for $P$ in $\mathfrak{P}(X)$ and for its (unique) extension on $\mathcal{B}_{c}(X)$. A function $f: X \rightarrow Y$ (where $Y$ is an other Polish space) is universally measurable or $\mathcal{B}_{c}(X)$-measurable [resp., Borel-measurable or $\mathcal{B}(X)$-measurable] if for all $B \in \mathcal{B}(Y), f^{-1}(B) \in \mathcal{B}_{c}(X)$ [resp., $f^{-1}(B) \in \mathcal{B}(X)$ ]. Similarly, we will speak of universally adapted or universally predictable (resp., Borel-adapted or Borel-predictable) processes. 
For a given $\mathcal{P} \subset \mathfrak{P}(X)$, a set $N \subset X$ is called a $\mathcal{P}$-polar if for all $P \in \mathcal{P}$, there exists some $A_{P} \in \mathcal{B}_{c}(X)$ such that $P\left(A_{P}\right)=0$ and $N \subset A_{P}$. We say that a property holds true $\mathcal{P}$-quasi-surely (q.s.), if it is true outside a $\mathcal{P}$-polar set. Finally, we say that a set is of $\mathcal{P}$-full measure if its complement is a $\mathcal{P}$-polar set.

1.2. Analytic sets. An analytic set of $X$ is the continuous image of a Polish space [see Aliprantis and Border (2006), Theorem 12.24, page 447]. We denote by $\mathcal{A}(X)$ the set of analytic sets of $X$ and recall some key properties that will often be used in the rest of the paper without further references [see also Bertsekas and Shreve (2004), Chapter 7, for more details on analytic sets]. The projection of an analytic set is an analytic set [see Bertsekas and Shreve (2004), Proposition 7.39, page 165] and the countable union, intersection or Cartesian product of analytic sets is an analytic set [see Bertsekas and Shreve (2004), Corollary 7.35.2, page 160, Proposition 7.38, page 165]. However, the complement of an analytic set does not need to be an analytic set. We denote by $\mathcal{C} A(X):=\{A \in X, X \backslash A \in \mathcal{A}(X)\}$ the set of all coanalytic sets of $X$. We have that [see Bertsekas and Shreve (2004), Proposition 7.36, page 161, Corollary 7.42.1, page 169]

$$
\mathcal{B}(X) \subset \mathcal{A}(X) \cap \mathcal{C} \mathcal{A}(X) \text { and } \mathcal{A}(X) \cup \mathcal{C} \mathcal{A}(X) \subset \mathcal{B}_{c}(X) .
$$

Now, for $D \in \mathcal{A}(X)$, a function $f: D \rightarrow \mathbb{R} \cup\{ \pm \infty\}$ is lower-semianalytic or l.s.a. [resp., upper-semianalytic or u.s.a.] on $X$ if $\{x \in X f(x)<c\} \in \mathcal{A}(X)$ [resp., $\{x \in X f(x)>c\} \in \mathcal{A}(X)]$ for all $c \in \mathbb{R}$. We denote by $\mathcal{L} S A(X)$ [resp., $\mathcal{U} S A(X)]$ the set of all 1.s.a. [resp., u.s.a.] functions on $X$. A function $f: X \rightarrow Y$ (where $Y$ is another Polish space) is analytically measurable if for all $B \in \mathcal{B}(Y), f^{-1}(B)$ belongs to the sigma-algebra generated by $\mathcal{A}(X)$. From (1), it is clear that if $f$ is 1.s.a. or u.s.a. or analytically measurable then $f$ is $\mathcal{B}_{c}(X)$-measurable; again this will be used through the paper without further references.

1.3. Measurable spaces, stochastic kernels and definition of $\mathcal{Q}^{T}$. We fix a time horizon $T \in \mathbb{N}$ and introduce a sequence $\left(\Omega_{t}\right)_{1 \leq t \leq T}$ of Polish spaces. We denote by $\Omega^{t}:=\Omega_{1} \times \cdots \times \Omega_{t}$, with the convention that $\Omega^{0}$ is reduced to a singleton. An element of $\Omega^{t}$ will be denoted by $\omega^{t}=\left(\omega_{1}, \ldots, \omega_{t}\right)=\left(\omega^{t-1}, \omega_{t}\right)$ for $\left(\omega_{1}, \ldots, \omega_{t}\right) \in \Omega_{1} \times \cdots \times \Omega_{t}$ and $\left(\omega^{t-1}, \omega_{t}\right) \in \Omega^{t-1} \times \Omega_{t}$ (to avoid heavy notation we drop the dependency in $\left.\omega_{0}\right)$. It is well known that $\mathcal{B}\left(\Omega^{t}\right)=\mathcal{B}\left(\Omega^{t-1}\right) \otimes \mathcal{B}\left(\Omega_{t}\right)$ [see Aliprantis and Border (2006), Theorem 4.44, page 149]. However, we have only that $\mathcal{B}_{c}\left(\Omega^{t-1}\right) \otimes \mathcal{B}_{c}\left(\Omega_{t}\right) \subset \mathcal{B}_{c}\left(\Omega^{t}\right)$, which makes the use of the projection theorem problematic and enlighten why analytic sets are introduced. For all $0 \leq t \leq T-1$, we denote by $\mathcal{S} K_{t+1}$ the set of universally measurable stochastic kernel on $\Omega_{t+1}$ given $\Omega^{t}$ [see Bertsekas and Shreve (2004), Definition 7.12, page 134, Lemma 7.28, page 174]. Fix some $1 \leq t \leq T, P_{t-1} \in \mathfrak{P}\left(\Omega^{t-1}\right)$ and $p_{t} \in \mathcal{S} K_{t}$. Using Fubini's theorem [see Bertsekas and Shreve (2004), Proposition 7.45, page 175], we set for all $A \in \mathcal{B}_{c}\left(\Omega^{t}\right)$

$$
P_{t-1} \otimes p_{t}(A):=\int_{\Omega^{t-1}} \int_{\Omega_{t}} 1_{A}\left(\omega^{t-1}, \omega_{t}\right) p_{t}\left(d \omega_{t}, \omega^{t-1}\right) P_{t-1}\left(d \omega^{t-1}\right) .
$$


For all $0 \leq t \leq T-1$, we consider the random sets $\mathcal{Q}_{t+1}: \Omega^{t} \rightarrow \mathfrak{P}\left(\Omega_{t+1}\right)$ : $\mathcal{Q}_{t+1}\left(\omega^{t}\right)$ can be seen as the set of possible models for the $t+1$ th period given the state $\omega^{t}$ until time $t$.

AsSUMPTION 1.1. For all $0 \leq t \leq T-1, \mathcal{Q}_{t+1}$ is a nonempty and convex valued random set such that

$$
\operatorname{Graph}\left(\mathcal{Q}_{t+1}\right)=\left\{\left(\omega^{t}, P\right), P \in \mathcal{Q}_{t+1}\left(\omega^{t}\right)\right\} \in \mathcal{A}\left(\Omega^{t} \times \mathfrak{P}\left(\Omega_{t+1}\right)\right) .
$$

From the Jankov-von Neumann theorem [see Bertsekas and Shreve (2004), Proposition 7.49, page 182], there exists some analytically measurable $q_{t+1}$ : $\Omega^{t} \rightarrow \mathfrak{P}\left(\Omega_{t+1}\right)$ such that for all $\omega^{t} \in \Omega^{t}, q_{t+1}\left(\cdot, \omega^{t}\right) \in \mathcal{Q}_{t+1}\left(\omega^{t}\right)$ [recall that for all $\left.\omega^{t} \in \Omega^{t}, \mathcal{Q}_{t+1}\left(\omega^{t}\right) \neq \varnothing\right]$. In other words, $q_{t+1} \in \mathcal{S} K_{t+1}$ is a universally measurable selector of $\mathcal{Q}_{t+1}$. For all $1 \leq t \leq T$, we define $\mathcal{Q}^{t} \subset \mathfrak{P}\left(\Omega^{t}\right)$ by

$$
\begin{aligned}
\mathcal{Q}^{t}:= & \left\{Q_{1} \otimes q_{2} \otimes \cdots \otimes q_{t}, Q_{1} \in \mathcal{Q}_{1}, q_{s+1} \in \mathcal{S} K_{s+1},\right. \\
& \left.q_{s+1}\left(\cdot, \omega^{s}\right) \in \mathcal{Q}_{s+1}\left(\omega^{s}\right) Q_{s} \text {-a.s. } \forall 1 \leq s \leq t-1\right\},
\end{aligned}
$$

where if $Q_{t}=Q_{1} \otimes q_{2} \otimes \cdots \otimes q_{t} \in \mathcal{Q}^{t}$ we write for any $2 \leq s \leq t Q_{s}:=Q_{1} \otimes$ $q_{2} \otimes \cdots \otimes q_{s}$ and $Q_{s} \in \mathcal{Q}^{s}$. For any fixed $P \in \mathcal{Q}^{T}, E_{P}$ denotes the expectation under $P$.

1.4. The traded assets and strategies. Let $S:=\left\{S_{t}, 0 \leq t \leq T\right\}$ be a universally adapted $d$-dimensional process where for $0 \leq t \leq T, S_{t}=\left(S_{t}^{i}\right)_{1 \leq i \leq d}$ represents the price of $d$ risky securities in the financial market in consideration. We make the following assumptions which were already stated in Nutz (2016).

Assumption 1.2. The process $S$ is Borel-adapted.

REMARK 1.3. If Assumption 1.2 is not postulated, we cannot obtain some crucial measurability properties [see Bouchard and Nutz (2015), Remark 4.4, Lemma 2.2 below as well as (26) and (27) and Bertsekas and Shreve (2004), Lemma 7.30(3), page 178]. Note that this assumption is not needed in the one period case.

Assumption 1.4. There exists some $0 \leq s<\infty$ such that $-s \leq S_{t}^{i}\left(\omega^{t}\right)<$ $+\infty$ for all $1 \leq i \leq d, \omega^{t} \in \Omega^{t}$ and $0 \leq t \leq T$.

Note that we can easily incorporate the case where $-s \leq S_{t}^{i}<+\infty$ only on a Borel $\mathcal{Q}^{T}$-full measure set. There exists also a riskless asset for which we assume a price constant equal to 1 , for sake of simplicity. Without this assumption, all the developments below could be carried out using discounted prices. The notation $\Delta S_{t}:=S_{t}-S_{t-1}$ will often be used. If $x, y \in \mathbb{R}^{d}$, then the concatenation $x y$ stands for their scalar product. The symbol $|\cdot|$ denotes the Euclidean norm on $\mathbb{R}^{d}$ (or 
on $\mathbb{R}$ ). Trading strategies are represented by $d$-dimensional universally predictable processes $\phi:=\left\{\phi_{t}, 1 \leq t \leq T\right\}$ where for all $1 \leq t \leq T, \phi_{t}=\left(\phi_{t}^{i}\right)_{1 \leq i \leq d}$ represents the investor's holdings in each of the $d$ assets at time $t$. The family of all such trading strategies is denoted by $\Phi$. We assume that trading is self-financing. As the riskless asset's price is constant equal to 1 , the value at time $t$ of a portfolio $\phi$ starting from initial capital $x \in \mathbb{R}$ is given by $V_{t}^{x, \phi}=x+\sum_{s=1}^{t} \phi_{s} \Delta S_{s}$.

From now on, the positive (resp., negative) part of some number or random variable $Y$ is denoted by $Y^{+}$(resp., $Y^{-}$). We will also write $f^{ \pm}(Y)$ for $(f(Y))^{ \pm}$ for any random variable $Y$ and (possibly random) function $f$.

\subsection{No arbitrage condition, risk preferences and main result.}

DEFINITION 1.5. The $N A\left(\mathcal{Q}^{T}\right)$ condition holds true if for $\phi \in \Phi, V_{T}^{0, \phi} \geq$ $0 \mathcal{Q}^{T}$-q.s. implies that $V_{T}^{0, \phi}=0 \mathcal{Q}^{T}$-q.s. [see also Bouchard and Nutz (2015), Definition 1.1].

DEFINITION 1.6. A random utility $U$ is a function defined on $\Omega^{T} \times(0, \infty)$ taking values in $\mathbb{R} \cup\{-\infty\}$ such that for every $x \in \mathbb{R}, U(\cdot, x)$ is $\mathcal{B}\left(\Omega^{T}\right)$-measurable and for every $\omega^{T} \in \Omega^{T}, U\left(\omega^{T}, \cdot\right)$ is proper, ${ }^{2}$ nondecreasing and concave on $(0,+\infty)$. We extend $U$ by (right) continuity in 0 and set $U(\cdot, x)=-\infty$ if $x<0$.

REMARK 1.7. Fix some $\omega^{T} \in \Omega^{T}$ and let $\operatorname{Dom} U\left(\omega^{T}, \cdot\right):=\{x \in \mathbb{R}$, $\left.U\left(\omega^{T}, x\right)>-\infty\right\}$ be the domain of $U\left(\omega^{T}, \cdot\right)$. Then $U\left(\omega^{T}, \cdot\right)$ is continuous on $\operatorname{Ri}\left(\operatorname{Dom} U\left(\omega^{T}, \cdot\right)\right)$, the relative interior of the domain of $U\left(\omega^{T}, \cdot\right)$ [see Rockafellar (1970), Theorem 10.1, page 82]. Note that if $U\left(\omega^{T}, \cdot\right)$ is improper then $U\left(\omega^{T}, \cdot\right)=$ $+\infty$ on $\operatorname{Ri}\left(\operatorname{Dom} U\left(\omega^{T}, \cdot\right)\right)$ and if $U\left(\omega^{T}, \cdot\right)$ is assumed to be upper semicontinuous (u.s.c. from now) then it is infinite on all $\mathbb{R}$ [see Rockafellar (1970), Theorem 7.2 and Corollary 7.2.1, page 53] which is a rather uninteresting case. Nevertheless, our results hold true for an improper u.s.c. function. Here, $U\left(\omega^{T}, \cdot\right)$ will not be assumed to be u.s.c. since Assumption 3.1 is postulated. Indeed it implies that $\operatorname{Dom} U\left(\omega^{T}, \cdot\right)=(0, \infty)$ if $\omega^{T} \in \Omega_{\text {Dom }}^{T}$ which is a Borel $\mathcal{Q}^{T}$-full measure set [see Lemma 3.2]. Then $U$ can be modified so that it remains Borel-measurable, that Dom $U\left(\omega^{T}, \cdot\right)=(0, \infty)$, and thus extending $U\left(\omega^{T}, \cdot\right)$ by continuity in 0 is enough to get an u.s.c. function for all $\omega^{T} \in \Omega^{T}$. If $\operatorname{Dom} U\left(\omega^{T}, \cdot\right)=(0, \infty)$ is not true on a Borel $\mathcal{Q}^{T}$-full measure set, then one cannot avoid the u.s.c. assumption: $U\left(\omega^{T}, \cdot\right)$ is continuous on $\operatorname{Ri}\left(\operatorname{Dom} U\left(\omega^{T}, \cdot\right)\right)=\left(m\left(\omega^{T}\right), \infty\right)$ and one needs to extend $U\left(\omega^{T}, \cdot\right)$ by (right)-continuity in $m\left(\omega^{T}\right)$ which might be strictly positive. This is the reason why in the dynamic programming part we force the value function to be u.s.c. on all $\Omega^{t}$ by taking their closure [see Lemma 3.18, (19) and (24)]. Note that we can easily include the case where $U\left(\omega^{T}, \cdot\right)$ is nondecreasing and concave only for $\omega^{T}$ in a Borel $\mathcal{Q}^{T}$-full measure set. We introduce the following notation.

\footnotetext{
${ }^{2}$ There exists $x \in(0,+\infty)$ such that $U\left(\omega^{T}, x\right)>-\infty$ and $U\left(\omega^{T}, x\right)<+\infty$ for all $x \in(0,+\infty)$.
} 
Definition 1.8. Fix some $x \geq 0$. For $P \in \mathfrak{P}\left(\Omega^{T}\right)$ fixed, we denote by $\Phi(x, P)$ the set of all strategies $\phi \in \Phi$ such that $V_{T}^{x, \phi}(\cdot) \geq 0 P$-a.s. and by $\Phi(x, U, P)$ the set of all strategies $\phi \in \Phi(x, P)$ such that either $E_{P} U^{+}\left(\cdot, V_{T}^{x, \phi}(\cdot)\right)<\infty$ or $E_{P} U^{-}\left(\cdot, V_{T}^{x, \phi}(\cdot)\right)<\infty$. Then

$$
\Phi\left(x, \mathcal{Q}^{T}\right):=\bigcap_{P \in \mathcal{Q}^{T}} \Phi(x, P) \quad \text { and } \quad \Phi\left(x, U, \mathcal{Q}^{T}\right):=\bigcap_{P \in \mathcal{Q}^{T}} \Phi(x, U, P) .
$$

Under $N A\left(\mathcal{Q}^{T}\right)$, if $\phi \in \Phi\left(x, \mathcal{Q}^{T}\right)$ then $P_{t}\left(V_{t}^{x, \phi}(\cdot) \geq 0\right)=1$ for all $P \in \mathcal{Q}^{t}$ and $1 \leq t \leq T$; see Lemma A.33. Note that in the definition of $\mathcal{H}_{x}$ in Nutz (2016) (see top of page 10), this intertemporal budget constraint was postulated. We now state our main concern.

DEFINITION 1.9. Let $x \geq 0$. The multiple-priors portfolio problem with initial wealth $x$ is

$$
u(x):=\sup _{\phi \in \Phi\left(x, U, \mathcal{Q}^{T}\right)} \inf _{P \in \mathcal{Q}^{T}} E_{P} U\left(\cdot, V_{T}^{x, \phi}(\cdot)\right)
$$

REMARK 1.10. We will use the convention $+\infty-\infty=+\infty$ throughout the paper. This choice is rather unnatural when studying maximisation problem. The reason for this is that we will use Bertsekas and Shreve (2004), Proposition 7.48, page 180 [which relies on Bertsekas and Shreve (2004), Lemma 7.30(4), page 177] for lower-semianalytic function where this convention is required.

We now present our main result under conditions which will be detailed in Section 3.

THEOREM 1.11. Assume that the $N A\left(\mathcal{Q}^{T}\right)$ condition and Assumptions 1.1, $1.2,1.4,3.1$ and 3.5 hold true. Let $x \geq 0$. Then there exists some optimal strategy $\phi^{*} \in \Phi\left(x, U, \mathcal{Q}^{T}\right)$ such that

$$
u(x)=\inf _{P \in \mathcal{Q}^{T}} E_{P} U\left(\cdot, V_{T}^{x, \phi^{*}}(\cdot)\right)<\infty .
$$

In Theorem 3.6, we will propose a fairly general set-up where Assumption 3.5 is satisfied.

2. No-arbitrage condition characterisation. We will often use the following one-period version of the no-arbitrage condition. For $\omega^{t} \in \Omega^{t}$ fixed, we say that $N A\left(\mathcal{Q}_{t+1}\left(\omega^{t}\right)\right)$ condition holds true if for all $h \in \mathbb{R}^{d}$

$$
h \Delta S_{t+1}\left(\omega^{t}, \cdot\right) \geq 0 \mathcal{Q}_{t+1}\left(\omega^{t}\right) \text {-q.s. } \Rightarrow h \Delta S_{t+1}\left(\omega^{t}, \cdot\right)=0 \mathcal{Q}_{t+1}\left(\omega^{t}\right) \text {-q.s. }
$$

We introduce the affine hull (denoted by Aff) of the (robust) conditional support of $\Delta S_{t+1}$. 
Definition 2.1. Let $0 \leq t \leq T-1$ be fixed. The random set $D^{t+1}: \Omega^{t} \rightarrow \mathbb{R}^{d}$ is defined as

$$
\begin{aligned}
D^{t+1}\left(\omega^{t}\right):= & \operatorname{Aff}\left(\bigcap \left\{A \subset \mathbb{R}^{d},\right.\right. \text { closed, } \\
& \left.\left.P_{t+1}\left(\Delta S_{t+1}\left(\omega^{t}, \cdot\right) \in A\right)=1, \forall P_{t+1} \in \mathcal{Q}_{t+1}\left(\omega^{t}\right)\right\}\right) .
\end{aligned}
$$

A strategy $\phi \in \Phi$ such that $\phi^{t+1}\left(\omega^{t}\right) \in D^{t+1}\left(\omega^{t}\right)$ have nice properties; see (6) and Lemma 3.11. If $D^{t+1}\left(\omega^{t}\right)=\mathbb{R}^{d}$ then, intuitively, there are no redundant assets for all model specifications. Otherwise, for any $\mathcal{B}_{c}\left(\Omega^{t}\right)$-measurable strategy $\phi_{t+1}$, one may always replace $\phi_{t+1}\left(\omega^{t}, \cdot\right)$ by its orthogonal projection $\phi_{t+1}^{\perp}\left(\omega^{t}, \cdot\right)$ on $D^{t+1}\left(\omega^{t}\right)$ without changing the portfolio value [see Remark 3.10 below and Nutz (2016), Lemma 2.6]. The following lemma establishes some important properties of $D^{t+1}$

LEMMA 2.2. Let Assumptions 1.1 and 1.2 hold true and $0 \leq t \leq T-1$ be fixed. Then $D^{t+1}$ is a nonempty, closed valued random set and $\operatorname{Graph}\left(D^{t+1}\right) \in$ $\mathcal{B}_{c}\left(\Omega^{t}\right) \otimes \mathcal{B}\left(\mathbb{R}^{d}\right)$.

PROOF. The proof uses similar arguments as in Rockafellar and Wets (1998) Theorem 14.8, page 648, Example 14.2, page 652, together with Bouchard and Nutz (2015) Lemma 4.3 and is thus omitted.

Similarly, as in Rásonyi and Stettner (2005) and Jacod and Shiryaev (1998) [see also Blanchard, Carassus and Rásonyi (2016)], we prove a "quantitative" characterisation of the $N A\left(\mathcal{Q}^{T}\right)$ condition.

Proposition 2.3. Assume that the $N A\left(\mathcal{Q}^{T}\right)$ condition and Assumptions 1.1, 1.2 hold true. Then for all $0 \leq t \leq T-1$, there exists some $\mathcal{Q}^{t}$-full measure set $\Omega_{N A}^{t} \in \mathcal{B}_{c}\left(\Omega^{t}\right)$ such that for all $\omega^{t} \in \Omega_{N A}^{t}, N A\left(\mathcal{Q}_{t+1}\left(\omega^{t}\right)\right)$ holds true, $D^{t+1}\left(\omega^{t}\right)$ is a vector space and there exists $\alpha_{t}\left(\omega^{t}\right)>0$ such that for all $h \in D^{t+1}\left(\omega^{t}\right)$ there exists $P_{h} \in \mathcal{Q}_{t+1}\left(\omega^{t}\right)$ satisfying

$$
P_{h}\left(\frac{h}{|h|} \Delta S_{t+1}\left(\omega^{t}, \cdot\right)<-\alpha_{t}\left(\omega^{t}\right)\right)>\alpha_{t}\left(\omega^{t}\right)
$$

We prove in Blanchard and Carassus (2017) that there is in fact an equivalence between the $N A\left(\mathcal{Q}^{T}\right)$ condition and (6). We also prove that $\omega^{t} \rightarrow \alpha_{t}\left(\omega^{t}\right)$ is $\mathcal{B}_{c}\left(\Omega^{t}\right)$ measurable.

Proof. Using Bouchard and Nutz (2015), Theorem 4.5, $N_{t}:=\left\{\omega^{t} \in \Omega^{t}\right.$, $N A\left(\mathcal{Q}_{t+1}\left(\omega^{t}\right)\right)$ fails $\} \in \mathcal{B}_{c}\left(\Omega^{t}\right)$ and $P\left(N_{t}\right)=1$ for all $P \in \mathcal{Q}^{t}$. So setting $\Omega_{N A}^{t}:=$ 
$\Omega^{t} \backslash N_{t}$, we get that (5) holds true for all $\omega^{t} \in \Omega_{N A}^{t}$. We fix some $\omega^{t} \in \Omega_{N A}^{t}$. If $h \in D^{t+1}\left(\omega^{t}\right)$, we have that

$$
h \Delta S_{t+1}\left(\omega^{t}, \cdot\right) \geq 0 \mathcal{Q}_{t+1}\left(\omega^{t}\right) \text {-q.s. } \Rightarrow h=0 .
$$

Indeed as $\omega^{t} \in \Omega_{N A}^{t}$, (5) together with Nutz (2016), Lemma 2.6, imply that $h \in$ $\left(D^{t+1}\left(\omega^{t}\right)\right)^{\perp}$ the orthogonal space of $D^{t+1}\left(\omega^{t}\right)$ and $h=0$. Therefore, for all $h \in$ $D^{t+1}\left(\omega^{t}\right), h \neq 0$, there exists $P_{h} \in \mathcal{Q}_{t+1}\left(\omega^{t}\right)$ such that $P_{h}\left(h \Delta S_{t+1}\left(\omega^{t}, \cdot\right) \geq 0\right)<1$.

Using a slight modification of Blanchard, Carassus and Rásonyi (2016), Lemma 3.5, we get that $0 \in D^{t+1}\left(\omega^{t}\right)$ [i.e., $D^{t+1}\left(\omega^{t}\right)$ is a vector space]. We introduce for $n \geq 1$,

$$
\begin{aligned}
A_{n}\left(\omega^{t}\right):= & \left\{h \in D^{t+1}\left(\omega^{t}\right),|h|=1\right. \\
& \left.P_{t+1}\left(h \Delta S_{t+1}\left(\omega^{t}, \cdot\right) \leq-\frac{1}{n}\right) \leq \frac{1}{n}, \forall P_{t+1} \in \mathcal{Q}_{t+1}\left(\omega^{t}\right)\right\}
\end{aligned}
$$

and we define $n_{0}\left(\omega^{t}\right):=\inf \left\{n \geq 1, A_{n}\left(\omega^{t}\right)=\varnothing\right\}$ with the convention that inf $\varnothing=$ $+\infty$. If $D^{t+1}\left(\omega^{t}\right)=\{0\}$, then $n_{0}\left(\omega^{t}\right)=1<\infty$. We assume now that $D^{t+1}\left(\omega^{t}\right) \neq$ $\{0\}$ and prove by contradiction that $n_{0}\left(\omega^{t}\right)<\infty$. Suppose that $n_{0}\left(\omega^{t}\right)=\infty$. For all $n \geq 1$, we get some $h_{n}\left(\omega^{t}\right) \in D^{t+1}\left(\omega^{t}\right)$ with $\left|h_{n}\left(\omega^{t}\right)\right|=1$ and such that for all $P_{t+1} \in \mathcal{Q}_{t+1}\left(\omega^{t}\right) P_{t+1}\left(h_{n}\left(\omega^{t}\right) \Delta S_{t+1}\left(\omega^{t}, \cdot\right) \leq-\frac{1}{n}\right) \leq \frac{1}{n}$. By passing to a sub-sequence we can assume that $h_{n}\left(\omega^{t}\right)$ tends to some $h^{*}\left(\omega^{t}\right) \in$ $D^{t+1}\left(\omega^{t}\right)$ with $\left|h^{*}\left(\omega^{t}\right)\right|=1$. Then $\left\{h^{*}\left(\omega^{t}\right) \Delta S_{t+1}\left(\omega^{t}, \cdot\right)<0\right\} \subset \liminf _{n} B_{n}\left(\omega^{t}\right)$, where $B_{n}\left(\omega^{t}\right):=\left\{h_{n}\left(\omega^{t}\right) \Delta S_{t+1}\left(\omega^{t}, \cdot\right) \leq-1 / n\right\}$. Fatou's lemma implies that for any $P_{t+1} \in \mathcal{Q}_{t+1}\left(\omega^{t}\right)$,

$$
P_{t+1}\left(h^{*}\left(\omega^{t}\right) \Delta S_{t+1}\left(\omega^{t}, \cdot\right)<0\right) \leq \liminf _{n} \int_{\Omega_{t+1}} 1_{B_{n}\left(\omega^{t}\right)}\left(\omega_{t+1}\right) P_{t+1}\left(d \omega_{t+1}\right)=0 .
$$

This implies that $P_{t+1}\left(h^{*}\left(\omega^{t}\right) \Delta S_{t+1}\left(\omega^{t}, \cdot\right) \geq 0\right)=1$ for all $P_{t+1} \in \mathcal{Q}_{t+1}\left(\omega^{t}\right)$ and $h^{*}\left(\omega^{t}\right)=0$ [see (7)], which contradicts $\left|h^{*}\left(\omega^{t}\right)\right|=1$. Thus $n_{0}\left(\omega^{t}\right)<\infty$. We set for $\omega^{t} \in \Omega_{N A}^{t}, \alpha_{t}\left(\omega^{t}\right):=\frac{1}{n_{0}\left(\omega^{t}\right)}, \alpha_{t} \in(0,1]$ and by definition of $A_{n_{0}\left(\omega^{t}\right)}\left(\omega^{t}\right),(6)$ holds true.

Finally, we introduce an alternative notion of no arbitrage, called strong no arbitrage.

DEFINITION 2.4. We say that the $s N A\left(\mathcal{Q}^{T}\right)$ condition holds true if for all $P \in \mathcal{Q}^{T}$ and $\phi \in \Phi, V_{T}^{0, \phi} \geq 0 P$-a.s. implies that $V_{T}^{0, \phi}=0 P$-a.s.

The $\operatorname{sNA}\left(\mathcal{Q}^{T}\right)$ condition holds true if the "classical" no-arbitrage condition in model $P, N A(P)$, holds true for all $P \in \mathcal{Q}^{T}$. Note that if $\mathcal{Q}^{T}=\{P\}$ then $s N A\left(\mathcal{Q}^{T}\right)=N A\left(\mathcal{Q}^{T}\right)=N A(P)$. Clearly, the $s N A\left(\mathcal{Q}^{T}\right)$ condition is stronger than the $N A\left(\mathcal{Q}^{T}\right)$ condition. 
As in Blanchard, Carassus and Rásonyi (2016), Definition 3.3, we introduce for all $P=P_{1} \otimes q_{2} \otimes \cdots \otimes q_{T} \in \mathcal{Q}^{T}$ and for all $1 \leq t \leq T-1$,

$$
D_{P}^{t+1}\left(\omega^{t}\right):=\operatorname{Aff}\left(\bigcap\left\{A \subset \mathbb{R}^{d}, \text { closed, } q_{t+1}\left(\Delta S_{t+1}\left(\omega^{t}, .\right) \in A, \omega^{t}\right)=1\right\}\right) .
$$

The case $t=0$ is obtained by replacing $q_{t+1}\left(\cdot, \omega^{t}\right)$ by $P_{1}(\cdot)$.

Proposition 2.5. Assume that the $\operatorname{sNA}\left(\mathcal{Q}^{T}\right)$ condition and Assumptions 1.1 and 1.2 hold true and let $0 \leq t \leq T-1$. Fix some $P=P_{1} \otimes q_{2} \otimes \cdots \otimes q_{T} \in$ $\mathcal{Q}^{T}$. Then there exists $\Omega_{P}^{t} \in \mathcal{B}\left(\Omega^{t}\right)$ with $P_{t}\left(\Omega_{P}^{t}\right)=1$ such that for all $\omega^{t} \in \Omega_{P}^{t}$, there exists $\alpha_{t}^{P}\left(\omega^{t}\right) \in(0,1]$ such that for all $h \in D_{P}^{t+1}\left(\omega^{t}\right), q_{t+1}\left(h \Delta S_{t+1}\left(\omega^{t}, \cdot\right) \leq\right.$ $\left.-\alpha_{t}^{P}\left(\omega^{t}\right)|h|, \omega^{t}\right) \geq \alpha_{t}^{P}\left(\omega^{t}\right)$. Furthermore, $\omega^{t} \rightarrow \alpha_{t}^{P}\left(\omega^{t}\right)$ is $\mathcal{B}\left(\Omega^{t}\right)$-measurable.

PROOF. This is a careful adaptation of Blanchard, Carassus and Rásonyi (2016), Proposition 3.7 since $\mathcal{B}_{c}\left(\Omega^{t}\right)$ is not a product sigma-algebra.

\section{Utility maximisation problem.}

ASSUMPTION 3.1. For all $r \in \mathbb{Q}, r>0 \sup _{P \in \mathcal{Q}^{T}} E_{P} U^{-}(\cdot, r)<+\infty$.

The proof of the following lemma follows directly from Rockafellar (1970), Theorem 10.1, page 82 .

LEMmA 3.2. Assume that Assumption 3.1 holds true. Then $\Omega_{\text {Dom }}^{T}:=$ $\{U(\cdot, r)>-\infty, \forall r \in \mathbb{Q}, r>0\} \in \mathcal{B}\left(\Omega^{T}\right)$ is a $\mathcal{Q}^{T}$-full measure set. For all $\omega^{T} \in \Omega_{\text {Dom }}^{T}, \operatorname{Ri}\left(\operatorname{Dom} U\left(\omega^{T}, \cdot\right)\right)=(0, \infty)$ and $U\left(\omega^{T}, \cdot\right)$ is continuous on $(0, \infty)$, right-continuous in 0 , and thus u.s.c. on $\mathbb{R}$.

REMARK 3.3. Assumption 3.1, which does not appear in the mono-prior case [see Blanchard, Carassus and Rásonyi (2016)], allows to work with countable supremum [see (18)] and to have value functions with "good" measurability properties; see also Remark 3.14. We will prove [see Proposition 3.27] that Assumption 3.1 is preserved through the dynamic programming procedure. Assumption 3.1 is superfluous in the case of nonrandom utility function. Indeed let $m:=\inf \{x \in$ $\mathbb{R}, U(x)>-\infty\} \geq 0$ and $\bar{U}(x)=U(x+m)$. Then $\operatorname{Ri}(\operatorname{Dom} \bar{U}(\cdot))=(0, \infty), \bar{U}$ satisfies Definition 1.6 and if $\bar{\phi}^{*}$ is a solution of (4) for $\bar{U}$ with an initial wealth $x$, then it will be a solution of (4) for $U$ starting from $x+m$. Assumption 3.1 is also useless in the one-period case.

EXAMPLE 3.4. We propose the following example where Assumption 3.1 holds true. Assume that there exists some $x_{0}>0$ such that $\sup _{P \in \mathcal{Q}^{T}} E_{P} U^{-}\left(\cdot, x_{0}\right)<\infty$. Assume also that there exists some functions $f_{1}, f_{2}$ : 
$(0,1] \rightarrow(0, \infty)$ as well as some nonnegative $\mathcal{B}_{c}\left(\Omega^{T}\right)$-measurable random variable $D$ verifying $\sup _{P \in \mathcal{Q}^{T}} E_{P} D(\cdot)<\infty$ such that for all $\omega^{T} \in \Omega^{T}, x \geq 0$, $0<\lambda \leq 1, U\left(\omega^{T}, \lambda x\right) \geq f_{1}(\lambda) U\left(\omega^{T}, x\right)-f_{2}(\lambda) D\left(\omega^{T}\right)$. This condition is a kind of elasticity assumption around zero. It is satisfied for example by the logarithm function. Fix some $r \in \mathbb{Q}, r>0$. If $r \geq x_{0}$, it is clear from Definition 1.6 that $\sup _{P \in \mathcal{Q}^{T}} E_{P} U^{-}(\cdot, r)<\infty$. If $r<x_{0}$, we have for all $\omega^{T} \in \Omega^{T}$, $U\left(\omega^{T}, r\right) \geq f_{1}\left(\frac{r}{x_{0}}\right) U\left(\omega^{T}, x_{0}\right)-f_{2}\left(\frac{r}{x_{0}}\right) D\left(\omega^{T}\right)$ and $\sup _{P \in \mathcal{Q}^{T}} E_{P} U^{-}(\cdot, r)<\infty$ follows immediately.

The following condition, together with Assumption 3.1, implies that if $\phi \in$ $\Phi\left(x, \mathcal{Q}^{T}\right)$ then $E_{P} U\left(\cdot, V_{T}^{x, \phi}(\cdot)\right)$ is well defined for all $P \in \mathcal{Q}^{T}$ [see Proposition 3.25]. It also allows us to work with auxiliary functions which play the role of properly integrable bounds for the value functions at each step [see (20), (27), (28) and (29)].

Assumption 3.5. We assume that

$$
\sup _{P \in \mathcal{Q}^{T}} \sup _{\phi \in \Phi(1, P)} E_{P} U^{+}\left(\cdot, V_{T}^{1, \phi}(\cdot)\right)<\infty .
$$

Assumption 3.5 is not easy to verify: we propose an application of Theorem 1.11 in the following fairly general set-up where Assumption 3.5 is automatically satisfied. We introduce for all $1 \leq t \leq T, r>0$,

$$
\begin{aligned}
\mathcal{W}_{t}^{r} & :=\left\{X: \Omega^{t} \rightarrow \mathbb{R} \cup\{ \pm \infty\}, \mathcal{B}\left(\Omega^{t}\right) \text {-measurable, } \sup _{P \in \mathcal{Q}^{t}} E_{P}|X|^{r}<\infty\right\}, \\
\mathcal{W}_{t} & :=\bigcap_{r>0} \mathcal{W}_{t}^{r} .
\end{aligned}
$$

In Denis, Hu and Peng (2011), Proposition 14, it is proved that $\mathcal{W}_{t}^{r}$ is a Banach space (up to the usual quotient identifying two random variables that are $\mathcal{Q}^{t}$-q.s. equal) for the norm $\|X\|:=\left(\sup _{P \in \mathcal{Q}^{t}} E_{P}|X|^{r}\right)^{\frac{1}{r}}$. Hence, the space $\mathcal{W}_{t}$ is the "natural" extension of the one introduced in the mono-prior classical case [see Carassus and Rásonyi (2016) or Blanchard, Carassus and Rásonyi (2016), (16)].

THEOREM 3.6. Assume that the $\operatorname{sA}\left(\mathcal{Q}^{T}\right)$ condition and Assumptions 1.1, 1.2, 1.4 and 3.1 hold true. Assume furthermore that $U^{+}(\cdot, 1), U^{-}\left(\cdot, \frac{1}{4}\right) \in \mathcal{W}_{T}$ and that for all $1 \leq t \leq T, P \in \mathcal{Q}^{t}, \Delta S_{t}, \frac{1}{\alpha_{t}^{P}} \in \mathcal{W}_{t}$ (recall Proposition 2.5 for the definition of $\left.\alpha_{t}^{P}\right)$. Let $x \geq 0$. Then there exists some optimal strategy $\phi^{*} \in \Phi\left(x, U, \mathcal{Q}^{T}\right)$ such that

$$
u(x)=\inf _{P \in \mathcal{Q}^{T}} E_{P} U\left(\cdot, V_{T}^{x, \phi^{*}}(\cdot)\right)<\infty .
$$


3.1. One period case. Let $(\bar{\Omega}, \mathcal{G})$ be a measurable space, $\mathfrak{P}(\bar{\Omega})$ the set of all probability measures on $\bar{\Omega}$ defined on $\mathcal{G}$ and $\mathcal{Q}$ a nonempty convex subset of $\mathfrak{P}(\bar{\Omega})$. Let $Y(\cdot):=\left(Y_{1}(\cdot), \ldots, Y_{d}(\cdot)\right)$ be a $\mathcal{G}$-measurable $\mathbb{R}^{d}$-valued random variable (which could represent the change of value of the price process).

Assumption 3.7. There exists some constant $0<b<\infty$ such that $Y_{i}(\cdot) \geq$ $-b$ for all $i=1, \ldots, d$.

Finally, as in Definition 2.1, $D \subset \mathbb{R}^{d}$ is the smallest affine subspace of $\mathbb{R}^{d}$ containing the support of the distribution of $Y(\cdot)$ under $P$ for all $P \in \mathcal{Q}$.

AsSumption 3.8. The set $D$ contains 0 ( $D$ is a nonempty vector subspace of $\mathbb{R}^{d}$ ).

The pendant of the $N A\left(\mathcal{Q}^{T}\right)$ condition in the one-period model is given by the following.

Assumption 3.9. There exists some constant $0<\alpha \leq 1$ such that for all $h \in D$ there exists $P_{h} \in \mathcal{Q}$ satisfying $P_{h}(h Y(\cdot) \leq-\alpha|h|) \geq \alpha$.

REMARK 3.10. Let $h \in \mathbb{R}^{d}$ and $h^{\prime} \in \mathbb{R}^{d}$ be the orthogonal projection of $h$ on $D$. Then $h-h^{\prime} \perp D$ hence

$$
\{Y(\cdot) \in D\} \subset\left\{\left(h-h^{\prime}\right) Y(\cdot)=0\right\}=\left\{h Y(\cdot)=h^{\prime} Y(\cdot)\right\} .
$$

By definition of $D$, we have $P(Y(\cdot) \in D)=1$ for all $P \in \mathcal{Q}$ and, therefore, $h Y=$ $h^{\prime} Y \mathcal{Q}$-q.s.

For $x \geq 0$ and $a \geq 0$, we define

$$
\begin{aligned}
\mathcal{H}_{x}^{a} & :=\left\{h \in \mathbb{R}^{d}, x+h Y \geq a \mathcal{Q} \text {-q.s. }\right\}, \\
D_{x} & :=\mathcal{H}_{x} \cap D \text { where } \mathcal{H}_{x}:=\mathcal{H}_{x}^{0} .
\end{aligned}
$$

LeMma 3.11. Assume that Assumption 3.9 holds true. Then for all $x \geq 0$, $D_{x} \subset B\left(0, \frac{x}{\alpha}\right)$ where $B\left(0, \frac{x}{\alpha}\right)=\left\{h \in \mathbb{R}^{d},|h| \leq \frac{x}{\alpha}\right\}$ and $D_{x}$ is a convex and compact subspace of $\mathbb{R}^{d}$.

PRoof. For $x \geq 0$, the convexity and the closedness of $D_{x}$ are clear. Let $h \in$ $D_{x}$ be fixed. Assume that $|h|>\frac{x}{\alpha}$, then from Assumption 3.9, there exists $P_{h} \in \mathcal{Q}$ such that $P_{h}(x+h Y(\cdot)<0) \geq P_{h}(h Y(\cdot) \leq-\alpha|h|) \geq \alpha>0$, a contradiction. The compactness of $D_{x}$ follows immediately. 
ASSUMPTION 3.12. We consider a function $V: \bar{\Omega} \times \mathbb{R} \rightarrow \mathbb{R} \cup\{ \pm \infty\}$ such that for every $x \in \mathbb{R}, V(\cdot, x): \bar{\Omega} \rightarrow \mathbb{R} \cup\{ \pm \infty\}$ is $\mathcal{G}$-measurable, for every $\omega \in \bar{\Omega}$, $V(\omega, \cdot): \mathbb{R} \rightarrow \mathbb{R} \cup\{ \pm \infty\}$ is nondecreasing, concave and u.s.c., and $V(\cdot, x)=-\infty$, for all $x<0$.

The reason for not excluding at this stage improper concave function is related to the multi-period case. Indeed if Assumption 3.9 is not verified, then $v$ [or $v^{\mathbb{Q}}$, $\left.\mathrm{Cl}\left(v^{\mathbb{Q}}\right)\right]$ might be equal to $+\infty$. So in the multi-period part, finding a version of the value function that is proper for all $\omega^{t}$ while preserving its measurability is challenging since $\Omega_{N A}^{t}$ (the set where Assumption 3.9 holds true, see Proposition 2.3) is only universally measurable. So here we do not assume that $V(\omega, \cdot)$ is proper but we will prove in Theorem 3.23 that the associated value function is finite. We also assume that $V(\omega, \cdot)$ is u.s.c. for all $\omega$; see Remark 1.7.

ASSUMPTION 3.13. For all $r \in \mathbb{Q}, r>0, \sup _{P \in \mathcal{Q}} E_{P} V^{-}(\cdot, r)<\infty$.

REMARK 3.14. This assumption is essential to prove in Theorem 3.23 that (14) holds true as it allows to prove that $\mathbb{Q}^{d}$ is dense in $\operatorname{Ri}\left(\left\{h \in \mathcal{H}_{x}\right.\right.$, $\left.\left.\inf _{P \in \mathcal{Q}} E V(\cdot, x+h Y(\cdot))>-\infty\right\}\right)$. Note that the one-period optimisation problem in (9) could be solved without Assumption 3.13 [see Remark 3.3].

The following lemma is similar to Lemma 3.2 [recall also Blanchard, Carassus and Rásonyi (2016), Lemma 7.12].

Lemma 3.15. Assume that Assumptions 3.12 and 3.13 hold true. Then $\Omega_{\text {Dom }}:=\{V(\cdot, r)>-\infty, \forall r \in \mathbb{Q}, r>0\} \in \mathcal{G}$ and $\Omega_{\text {Dom }}$ is $\mathcal{Q}$-full measure set on which $\operatorname{Ri}(\operatorname{Dom} V(\omega, \cdot))=(0, \infty)$, and thus $V(\omega, \cdot)$ is continuous on $(0, \infty)$. Moreover, $V(\omega, \cdot)$ is right-continuous in 0 for all $\omega \in \bar{\Omega}$.

Our main concern in the one period case is the following optimisation problem:

$$
v(x):= \begin{cases}\sup _{h \in \mathcal{H}_{x}} \inf _{P \in \mathcal{Q}} E_{P} V(\cdot, x+h Y(\cdot)) & \text { if } x \geq 0, \\ -\infty & \text { otherwise. }\end{cases}
$$

We use the convention $\infty-\infty=\infty$ [see Remark 1.10], but we will see in Lemma 3.21 that under appropriate assumptions, $E_{P} V(\cdot, x+h Y(\cdot))$ is well defined. Note also that for $x \geq 0$ [see Remark 3.10]

$$
v(x)=\sup _{h \in D_{x}} \inf _{P \in \mathcal{Q}} E_{P} V(\cdot, x+h Y(\cdot)) .
$$

We present now some integrability assumptions on $V^{+}$which allow to assert that there exists some optimal solution for (9). 
Assumption 3.16. For every $P \in \mathcal{Q}, h \in \mathcal{H}_{1}, E_{P} V^{+}(\cdot, 1+h Y(\cdot))<\infty$.

REMARK 3.17. If Assumption 3.16 is not true, Nutz (2016), Example 2.3 shows that one can find a counter-example where $v(x)<\infty$ but the supremum is not attained in (9). So one cannot use the "natural" extension of the mono-prior approach, which should be that there exists some $P \in \mathcal{Q}$ such that $E_{P} V^{+}(\cdot, 1+h Y(\cdot))<\infty$ for all $h \in \mathcal{H}_{1}$ [see Blanchard, Carassus and Rásonyi (2016), Assumption 5.9].

We define now

$$
v^{\mathbb{Q}}(x):= \begin{cases}\sup _{h \in \mathcal{H}_{x} \cap \mathbb{Q}^{d}} \inf _{P \in \mathcal{Q}} E_{P} V(\cdot, x+h Y(\cdot)) & \text { if } x \geq 0, \\ -\infty & \text { otherwise }\end{cases}
$$

Finally, we introduce the closure of $v^{\mathbb{Q}}$ denoted by $\mathrm{Cl}\left(v^{\mathbb{Q}}\right)$ which is the smallest u.s.c. function $w: \mathbb{R} \rightarrow \mathbb{R} \cup\{ \pm \infty\}$ such that $w \geq v^{\mathbb{Q}}$. We will show in Theorem 3.23 that $v(x)=v^{\mathbb{Q}}(x)=\mathrm{Cl}\left(v^{\mathbb{Q}}\right)(x)$, which allows in the multiperiod case [see (18)] to work with a countable supremum (for measurability issues) and an u.s.c. value function [see Remark 1.7]. But first we provide two lemmata which are stated under Assumption 3.12 only. They will be used in the multi-period part to prove that the value function is u.s.c., concave [see (24) and (25)] and dominated [see (28)] for all $\omega^{t}$. This avoids difficult measurability issues when proving (26) and (27) coming from full-measure sets which are not Borel and on which Assumptions 3.8, 3.9, 3.13 and 3.16 hold true. This can be seen, for example, in the beginning of the proof of Proposition 3.30 where we need to apply Lemma 3.18 using only Assumption 3.12.

Lemma 3.18. Assume that Assumption 3.12 holds true. Then $v, v^{\mathbb{Q}}$ and $\mathrm{Cl}\left(v^{\mathbb{Q}}\right)$ are concave and nondecreasing on $\mathbb{R}$ and $\mathrm{Cl}\left(v^{\mathbb{Q}}\right)(x)=\lim _{\delta \rightarrow 0} v^{\mathbb{Q}>0}(x+\delta)$.

Proof. As $V$ is nondecreasing [see Assumption 3.12], $v$ and $v^{\mathbb{Q}}$ are clearly nondecreasing. The proof of the concavity of $v$ or $v^{\mathbb{Q}}$ relies on a midpoint concavity argument and on the Ostrowski theorem; see Donoghue (1969), page 12. It is very similar to Rásonyi and Stettner (2006), Proposition 2 or Nutz (2016), Lemma 3.5, and thus omitted. Using Rockafellar and Wets (1998), Proposition 2.32 , page 57 , we obtain that $\mathrm{Cl}\left(v^{\mathbb{Q}}\right)$ is concave on $\mathbb{R}$. Then using, for example, Rockafellar and Wets (1998), 1(7), page 14, we get that for all $x \in \mathbb{R}$, $\mathrm{Cl}\left(v^{\mathbb{Q}}\right)(x)=\lim _{\delta \rightarrow 0} \sup _{|y-x|<\delta} v^{\mathbb{Q}}(y)=\lim _{\delta \rightarrow 0} v_{\delta>0} \mathbb{Q}(x+\delta)$ and the proof is completed.

Let $x \geq 0$ and $P \in \mathcal{Q}$ be fixed. We introduce $H_{x}(P):=\left\{h \in \mathbb{R}^{d}, x+h Y \geq\right.$ $0 P$-a.s. . Note that $\mathcal{H}_{x}=\bigcap_{P \in \mathcal{Q}} H_{x}(P)$ [see (8)]. 
LEMMA 3.19. Assume that Assumption 3.12 holds true. Let I $: \bar{\Omega} \times \mathbb{R} \rightarrow$ $[0, \infty]$ be a function such that for all $x \in \mathbb{R}$ and $h \in \mathbb{R}^{d}, I(\cdot, x+h Y(\cdot))$ is $\mathcal{G}$ measurable, $I(\omega, \cdot)$ is nondecreasing and nonnegative for all $\omega \in \bar{\Omega}$ and $V \leq I$. Set

$$
i(x):=1_{[0, \infty)}(x) \sup _{h \in \mathbb{R}^{d}} \sup _{P \in \mathcal{Q}} 1_{H_{x}(P)}(h) E_{P} I(\cdot, x+h Y(\cdot)) .
$$

Then $i$ is nondecreasing, nonnegative on $\mathbb{R}$ and $\mathrm{Cl}\left(v^{\mathbb{Q}}\right)(x) \leq i(x+1)$ for all $x \in \mathbb{R}$.

ProOF. Since $I(\cdot, x+h Y(\cdot))$ is $\mathcal{G}$-measurable for all $x \in \mathbb{R}$ and $I \geq 0$, the integral in the definition of $i$ is well defined (potentially equals to $+\infty$ ). It is clear that $i$ is nondecreasing and nonnegative on $\mathbb{R}$. As $V \leq I$ and $\mathcal{H}_{x} \subset H_{x}(P)$ if $P \in$ $\mathcal{Q}$, it is clear that $v^{\mathbb{Q}}(x) \leq i(x)$ for $x \geq 0$. And since $v^{\mathbb{Q}}(x)=-\infty<i(x)=0$ for $x<0, v^{\mathbb{Q}} \leq i$ on $\mathbb{R}$ (note that $v \leq i$ on $\mathbb{R}$ for the same reasons). Applying Lemma 3.18, $\mathrm{Cl}\left(v^{\mathbb{Q}}\right)(x) \leq v^{\mathbb{Q}}(x+1) \leq i(x+1)$ for all $x \in \mathbb{R}$.

Proposition 3.20. Assume that Assumptions 3.12 and 3.13 hold true. Then there exists some nonnegative $\mathcal{G}$-measurable random variable $C$ such that $\sup _{P \in \mathcal{Q}} E_{P}(C)<\infty$ and for all $\omega \in \Omega_{\text {Dom }}$ [see Lemma 3.15], $\lambda \geq 1, x \in \mathbb{R}$ we have

$$
V(\omega, \lambda x) \leq 2 \lambda\left(V\left(\omega, x+\frac{1}{2}\right)+C(\omega)\right) .
$$

PROOF. We use similar arguments as Rásonyi and Stettner (2006), Lemma 2. It is clear that (11) is true if $x<0$. We fix $\omega \in \Omega_{\text {Dom }}, x \geq \frac{1}{2}$ and $\lambda \geq 1$. Then $\operatorname{Ri}(\operatorname{Dom} V(\omega, \cdot))=(0, \infty)$ [recall Lemma 3.15]. We assume first that there exists some $x_{0} \in \operatorname{Dom} V(\omega, \cdot)$ such that $V\left(\omega, x_{0}\right)<\infty$. Since $V(\omega, \cdot)$ is u.s.c. and concave, using similar arguments as in Rockafellar (1970), Corollary 7.2.1, page 53, we get that $V(\omega, \cdot)<\infty$ on $\mathbb{R}$. Using the fact that $V(\omega, \cdot)$ is concave and nondecreasing, we get that (recall that $x \geq \frac{1}{2}$ )

$$
\begin{aligned}
V(\omega, \lambda x) & \leq V(\omega, x)+\frac{V(\omega, x)-V\left(\omega, \frac{1}{4}\right)}{x-\frac{1}{4}}(\lambda-1) x \\
& \leq V(\omega, x)+2(\lambda-1)\left(V(\omega, x)+V^{-}\left(\omega, \frac{1}{4}\right)\right) \\
& \leq V(\omega, x)+2\left(\lambda-\frac{1}{2}\right)\left(V(\omega, x)+V^{-}\left(\omega, \frac{1}{4}\right)\right)+V^{-}\left(\omega, \frac{1}{4}\right) \\
& \leq 2 \lambda\left(V(\omega, x)+V^{-}\left(\omega, \frac{1}{4}\right)\right) \\
& \leq 2 \lambda\left(V\left(\omega, x+\frac{1}{2}\right)+V^{-}\left(\omega, \frac{1}{4}\right)\right) .
\end{aligned}
$$


Fix now $0 \leq x \leq \frac{1}{2}$ and $\lambda \geq 1$. Using again that $V(\omega, \cdot)$ is nondecreasing and (12), $V(\omega, \lambda x) \leq V\left(\omega, \lambda\left(x+\frac{1}{2}\right)\right) \leq 2 \lambda\left(V\left(\omega, x+\frac{1}{2}\right)+V^{-}\left(\omega, \frac{1}{4}\right)\right)$, and Proposition 3.20 is proved setting $C(\omega)=V^{-}\left(\omega, \frac{1}{4}\right)$ [recall Assumption 3.13] when there exists some $x_{0} \in \operatorname{Dom} V(\omega, \cdot)$ such that $V\left(\omega, x_{0}\right)<\infty$. Now, if this is not the case, $V(\omega, x)=\infty$ for all $x \in \operatorname{Dom} V(\omega, \cdot), C(\omega)=V^{-}\left(\omega, \frac{1}{4}\right)=0$ and (11) also holds true for all $x \geq 0$.

LEMMA 3.21. Assume that Assumptions 3.8, 3.9, 3.12, 3.13 and 3.16 hold true. Then there exists a nonnegative $\mathcal{G}$-measurable $L$ such that for all $P \in \mathcal{Q}$, $E_{P}(L)<\infty$ and for all $x \geq 0$ and $h \in \mathcal{H}_{x}, V^{+}(\cdot, x+h Y(\cdot)) \leq(4 x+1) L(\cdot)$ Q-q.s.

PROOF. The proof is a slight adaptation of the one of Blanchard, Carassus and Rásonyi (2016), Lemma 5.11 [see also Nutz (2016), Lemma 2.8] and is thus omitted. Note that the function $L$ is the one defined in Blanchard, Carassus and Rásonyi (2016), Lemma 5.11.

LeMmA 3.22. Assume that Assumptions 3.8, 3.9, 3.12, 3.13 and 3.16 hold true. Let $\mathcal{H}$ be the set valued function that assigns to each $x \geq 0$ the set $\mathcal{H}_{x}$. Then $\operatorname{Graph}(\mathcal{H})=\left\{(x, h) \in[0,+\infty) \times \mathbb{R}^{d}, h \in \mathcal{H}_{x}\right\}$ is a closed and convex subset of $\mathbb{R} \times \mathbb{R}^{d}$. Let $\psi: \mathbb{R} \times \mathbb{R}^{d} \rightarrow \mathbb{R} \cup\{ \pm \infty\}$ be defined by

$$
\psi(x, h):= \begin{cases}\inf _{P \in \mathcal{Q}} E_{P} V(\cdot, x+h Y(\cdot)) & \text { if }(x, h) \in \operatorname{Graph}(\mathcal{H}), \\ -\infty & \text { otherwise. }\end{cases}
$$

Then $\psi$ is u.s.c. and concave on $\mathbb{R} \times \mathbb{R}^{d}, \psi<+\infty$ on $\operatorname{Graph}(\mathcal{H})$ and $\psi(x, 0)>$ $-\infty$ for all $x>0$.

Proof. For all $P \in \mathcal{Q}$, we define $\psi_{P}: \mathbb{R} \times \mathbb{R}^{d} \rightarrow \mathbb{R} \cup\{ \pm \infty\}$ by $\psi_{P}(x, h)=$ $E_{P} V(\cdot, x+h Y(\cdot))$ if $(x, h) \in \operatorname{Graph}(\mathcal{H})$ and $-\infty$ otherwise. As in Blanchard, Carassus and Rásonyi (2016), Lemma 5.12, $\operatorname{Graph}(\mathcal{H})$ is a closed convex subset of $\mathbb{R} \times \mathbb{R}^{d}, \psi_{P}$ is u.s.c. on $\mathbb{R} \times \mathbb{R}^{d}$ and $\psi_{P}<\infty$ on $\operatorname{Graph}(\mathcal{H})$ for all $P \in \mathcal{Q}$. Furthermore, the concavity of $\psi_{P}$ follows immediately from the one of $V$. The function $\psi=\inf _{P \in \mathcal{Q}} \psi_{P}$ is then u.s.c. and concave. As $\psi_{P}<\infty$ on $\operatorname{Graph}(\mathcal{H})$ for all $P \in \mathcal{Q}$, it is clear that $\psi<+\infty$ on $\operatorname{Graph}(\mathcal{H})$. Finally, let $x>0$ be fixed and $r \in \mathbb{Q}$ be such that $r<x$, then we have $-\infty<\psi(r, 0) \leq \psi(x, 0)$; see Assumptions 3.12 and 3.13 .

We are now able to state the main result of this section.

THEOREM 3.23. Assume that Assumptions 3.7, 3.8, 3.9, 3.12, 3.13 and 3.16 hold true. Then for all $x \geq 0, v(x)<\infty$ and there exists some optimal strategy $\widehat{h} \in D_{x}$ such that

$$
v(x)=\inf _{P \in \mathcal{Q}} E_{P}(V(\cdot, x+\widehat{h} Y(\cdot)))
$$


Moreover $v$ is u.s.c., concave, nondecreasing and $\operatorname{Dom} v=(0, \infty)$. For all $x \in \mathbb{R}$,

$$
v(x)=v^{\mathbb{Q}}(x)=\operatorname{Cl}\left(v^{\mathbb{Q}}\right)(x) .
$$

Proof. Let $x \geq 0$ be fixed. Fix some $P \in \mathcal{Q}$. Using Lemma 3.21, we have that $E_{P} V(\cdot, x+h Y(\cdot)) \leq E_{P} V^{+}(\cdot, x+h Y(\cdot)) \leq(4 x+1) E_{P} L(\cdot)<\infty$, for all $h \in \mathcal{H}_{x}$. Thus $v(x)<\infty$. Now if $x>0, v(x) \geq \psi(x, 0)>-\infty$, see Lemma 3.22. Using Lemma 3.18, $v$ is concave and nondecreasing. Thus $v$ is continuous on $(0, \infty)$.

From Lemma 3.22, $h \rightarrow \psi(x, h)$ is u.s.c. on $\mathbb{R}^{d}$, and thus on $D_{x}$ [recall that $D_{x}$ is closed and use Blanchard, Carassus and Rásonyi (2016), Lemma 7.11]. Since $D_{x}$ is compact [see Lemma 3.11], recalling (10) and applying Aliprantis and Border (2006), Theorem 2.43, page 44, we find that there exists some $\widehat{h} \in D_{x}$ such that (13) holds true.

We prove now that $v$ is u.s.c. in 0 (the proof works as well for all $x^{*} \geq$ $0)$. Let $\left(x_{n}\right)_{n \geq 0}$ be a sequence of nonnegative numbers converging to 0 . Let $\widehat{h}_{n} \in D_{x_{n}}$ be the optimal strategies associated to $x_{n}$ in (13). Let $\left(n_{k}\right)_{k \geq 1}$ be a subsequence such that $\limsup _{n \rightarrow \infty} v\left(x_{n}\right)=\lim _{k \rightarrow \infty} v\left(x_{n_{k}}\right)$. Using Lemma 3.11, $\left|\widehat{h}_{n_{k}}\right| \leq x_{n_{k}} / \alpha \leq 1 / \alpha$ for $k$ big enough. So we can extract a subsequence, that we still denote by $\left(n_{k}\right)_{k \geq 1}$, such that there exists some $\underline{h}^{*}$ with $\widehat{h}_{n_{k}} \rightarrow \underline{h}^{*}$. As $\left(x_{n_{k}}, \hat{h}_{n_{k}}\right)_{k \geq 1} \in \operatorname{Graph}(\mathcal{H})$ which is a closed subset of $\mathbb{R} \times \mathbb{R}^{d}$ [see Lemma 3.22], $\underline{h}^{*} \in \mathcal{H}_{0}$. Thus using that $\psi$ is u.s.c., we get that

$$
\begin{aligned}
\limsup _{n \rightarrow \infty} v\left(x_{n}\right) & =\lim _{k \rightarrow \infty} \inf _{P \in \mathcal{Q}} E_{P} V\left(\cdot, x_{n_{k}}+\widehat{h}_{n_{k}} Y(\cdot)\right)=\lim _{k \rightarrow \infty} \psi\left(x_{n_{k}}, h_{n_{k}}\right) \\
& \leq \psi\left(0, \underline{h}^{*}\right)=\inf _{P \in \mathcal{Q}} E_{P} V\left(\cdot, \underline{h}^{*} Y(\cdot)\right) \leq v(0) .
\end{aligned}
$$

For $x<0$, all the equalities in (14) are trivial. We prove the first equality in (14) for $x \geq 0$ fixed. We start with the case $x=0$. If $Y=0 \mathcal{Q}$-q.s., then the first equality is trivial. If $Y \neq 0 \mathcal{Q}$-q.s., then it is clear that $D_{0}=\{0\}$ [recall Assumption 3.8] and the first equality in (14) is true again. We assume now that $x>0$. From Lemma 3.22, $\psi_{x}: h \rightarrow \psi(x, h)$ is concave, $0 \in \operatorname{Dom} \psi_{x}$. Thus $\operatorname{Ri}\left(\operatorname{Dom} \psi_{x}\right) \neq \varnothing$ [see Rockafellar (1970), Theorem 6.2, page 45] and we can apply Lemma A.32. Assume for a moment that we have proved that $\mathbb{Q}^{d}$ is dense in $\operatorname{Ri}\left(\operatorname{Dom} \psi_{x}\right)$. As $\psi_{x}$ is continuous on $\operatorname{Ri}\left(\operatorname{Dom} \psi_{x}\right.$ ) (recall that $\psi_{x}$ is concave), we obtain that

$$
\begin{aligned}
v(x) & =\sup _{h \in \mathcal{H}_{x}} \psi_{x}(h)=\sup _{h \in \operatorname{Dom} \psi_{x}} \psi_{x}(h)=\sup _{h \in \operatorname{Ri}\left(\operatorname{Dom} \psi_{x}\right)} \psi_{x}(h) \\
& =\sup _{h \in \operatorname{Ri}\left(\operatorname{Dom} \psi_{x}\right) \cap \mathbb{Q}^{d}} \psi_{x}(h) \leq \sup _{h \in \mathcal{H}_{x} \cap \mathbb{Q}^{d}} \psi_{x}(h)=v^{\mathbb{Q}}(x),
\end{aligned}
$$

since $\operatorname{Ri}\left(\operatorname{Dom} \psi_{x}\right) \subset \mathcal{H}_{x}$ and the first equality in (14) is proved. It remains to prove that $\mathbb{Q}^{d}$ is dense in $\operatorname{Ri}\left(\operatorname{Dom} \psi_{x}\right)$. Fix some $h \in \operatorname{Ri}\left(\mathcal{H}_{x}\right)$. From Lemma A.31, there is some $r \in \mathbb{Q}, r>0$ such that $h \in \mathcal{H}_{x}^{r}$. Using Lemma 3.22, we obtain that 
$\psi_{x}(h) \geq \psi(r, 0)>-\infty$ thus $h \in \operatorname{Dom} \psi_{x}$ and $\operatorname{Ri}\left(\mathcal{H}_{x}\right) \subset \operatorname{Dom} \psi_{x}$. Recalling that $0 \in \operatorname{Dom} \psi_{x}$ and that $\operatorname{Ri}\left(\mathcal{H}_{x}\right)$ is an open set in $\mathbb{R}^{d}$ [see Lemma A.31] we obtain that $\operatorname{Aff}\left(\operatorname{Dom} \psi_{x}\right)=\mathbb{R}^{d}$. Then $\operatorname{Ri}\left(\operatorname{Dom} \psi_{x}\right)$ is an open set in $\mathbb{R}^{d}$ and the fact that $\mathbb{Q}^{d}$ is dense in $\operatorname{Ri}\left(\operatorname{Dom} \psi_{x}\right)$ follows easily.

The second equality in (14) follows immediately: $v^{\mathbb{Q}}(x)=v(x)$ for all $x \geq 0$ and $v$ is u.s.c. on $[0, \infty)$, thus $\operatorname{Cl}\left(v^{\mathbb{Q}}\right)(x)=v^{\mathbb{Q}}(x)$ for all $x \geq 0$.

\subsection{Multi-period case.}

PROPOSITION 3.24. Assume that Assumption 3.1 holds true. Then there exists a nonnegative, $\mathcal{B}\left(\Omega^{T}\right)$-measurable random variable $C_{T}$ such that $\sup _{P \in \mathcal{Q}^{T}} E_{P}\left(C_{T}\right)<\infty$ and for all $\omega^{T} \in \Omega_{\text {Dom }}^{T}$ [recall Lemma 3.2], $\lambda \geq 1$ and $x \in \mathbb{R}$, we have

$$
\begin{gathered}
U\left(\omega^{T}, \lambda x\right) \leq 2 \lambda\left(U\left(\omega^{T}, x+\frac{1}{2}\right)+C_{T}\left(\omega^{T}\right)\right), \\
U^{+}\left(\omega^{T}, \lambda x\right) \leq 2 \lambda\left(U^{+}\left(\omega^{T}, x+\frac{1}{2}\right)+C_{T}\left(\omega^{T}\right)\right) .
\end{gathered}
$$

Proof. This is just Proposition 3.20 for $V=U$ and $\mathcal{G}=\mathcal{B}\left(\Omega^{T}\right)$, recaling Lemma 3.2 and setting $C_{T}(\cdot)=U^{-}\left(\cdot, \frac{1}{4}\right)$. The second inequality follows immediately since $C_{T}$ is nonnegative.

Proposition 3.25. Let Assumptions 3.1 and 3.5 hold true and fix some $x \geq 0$. Then

$$
M_{x}:=\sup _{P \in \mathcal{Q}^{T}} \sup _{\phi \in \phi(x, P)} E_{P} U^{+}\left(\cdot, V_{T}^{x, \phi}(\cdot)\right)<\infty .
$$

Moreover, $\Phi(x, U, P)=\Phi(x, P)$ for all $P \in \mathcal{Q}^{T}$, and thus $\Phi\left(x, U, \mathcal{Q}^{T}\right)=$ $\Phi\left(x, \mathcal{Q}^{T}\right)$.

Proof. Fix some $P \in \mathcal{Q}^{T}$. From Assumption 3.5, we know that $\Phi(1, P)=$ $\Phi(1, U, P)$ and $M_{1}<\infty$. Let $x \geq 0$ and $\phi \in \Phi(x, P)$ be fixed. If $x \leq 1$, then $V_{T}^{x, \phi} \leq V_{T}^{1, \phi}$, so from Definition 1.6 we get that $M_{x} \leq M_{1}<\infty$ and $\Phi(x, P)=$ $\Phi(x, U, P)$. If $x \geq 1$, from Proposition 3.24 we get that for all $\omega^{T} \in \Omega_{\text {Dom }}^{T}$

$$
\begin{aligned}
U^{+}\left(\omega^{T}, V_{T}^{x, \phi}\left(\omega^{T}\right)\right) & =U^{+}\left(\omega^{T}, 2 x\left(\frac{1}{2}+\sum_{t=1}^{T} \frac{\phi_{t}\left(\omega^{t-1}\right)}{2 x} \Delta S_{t}\left(\omega^{t}\right)\right)\right) \\
& \leq 4 x\left(U^{+}\left(\omega^{T}, V_{T}^{1, \frac{\phi}{2 x}}\left(\omega^{T}\right)\right)+C_{T}\left(\omega^{T}\right)\right) .
\end{aligned}
$$

As $\frac{\phi}{2 x} \in \Phi\left(\frac{1}{2}, P\right) \subset \Phi(1, P)=\Phi(1, U, P)$, we get that $M_{x} \leq 4 x\left(M_{1}+\right.$ $\left.\sup _{P \in \mathcal{Q}^{T}} E_{P} C_{T}\right)<\infty$, see Proposition 3.24. Thus $\Phi(x, P)=\Phi(x, U, P)$ and the last assertion follows from (3). 
We introduce now the dynamic programming procedure. First, we set for all $t \in\{0, \ldots, T-1\}, \omega^{t} \in \Omega^{t}, P \in \mathfrak{P}\left(\Omega_{t+1}\right)$ and $x \geq 0$ :

$$
\begin{aligned}
H_{x}^{t+1}\left(\omega^{t}, P\right) & :=\left\{h \in \mathbb{R}^{d}, x+h \Delta S_{t+1}\left(\omega^{t}, \cdot\right) \geq 0 P \text {-a.s. }\right\} \\
\mathcal{H}_{x}^{t+1}\left(\omega^{t}\right) & :=\left\{h \in \mathbb{R}^{d}, x+h \Delta S_{t+1}\left(\omega^{t}, \cdot\right) \geq 0 \mathcal{Q}_{t+1}\left(\omega^{t}\right) \text {-q.s. }\right\}, \\
\mathcal{D}_{x}^{t+1}\left(\omega^{t}\right) & :=\mathcal{H}_{x}^{t+1}\left(\omega^{t}\right) \cap D^{t+1}\left(\omega^{t}\right),
\end{aligned}
$$

where $D^{t+1}$ was introduced in Definition 2.1. For all $t \in\{0, \ldots, T-1\}, \omega^{t} \in \Omega^{t}$, $P \in \mathfrak{P}\left(\Omega_{t+1}\right)$ and $x<0$, we set $H_{x}^{t+1}\left(\omega^{t}, P\right)=\mathcal{H}_{x}^{t+1}\left(\omega^{t}\right)=\varnothing$. We introduce now the value functions $U_{t}$ from $\Omega^{t} \times \mathbb{R} \rightarrow \mathbb{R} \cup\{ \pm \infty\}$ for all $t \in\{0, \ldots, T\}$. To do that, we define the closure of a random function $F: \Omega^{t} \times \mathbb{R} \rightarrow \mathbb{R} \cup\{ \pm \infty\}$. Fix $\omega^{t} \in \Omega^{t}$, then $x \rightarrow F_{\omega^{t}}(x):=F\left(\omega^{t}, x\right)$ is a real-valued function and its closure is denoted by $\mathrm{Cl}\left(F_{\omega^{t}}\right)$. Now $\mathrm{Cl}(F): \Omega^{t} \times \mathbb{R} \rightarrow \mathbb{R} \cup\{ \pm \infty\}$ is defined by $\mathrm{Cl}(F)\left(\omega^{t}, x\right):=$ $\mathrm{Cl}\left(F_{\omega^{t}}\right)(x)$. For $0 \leq t \leq T$, we set for all $x \in \mathbb{R}$ and $\omega^{t} \in \Omega^{t}$

$$
\mathcal{U}_{T}\left(\omega^{T}, x\right):=U\left(\omega^{T}, x\right) 1_{\Omega_{\mathrm{Dom}}^{T} \times[0, \infty) \cup \Omega^{T} \times(-\infty, 0)}\left(\omega^{T}, x\right)
$$

$$
\mathcal{U}_{t}\left(\omega^{t}, x\right):=\left\{\begin{aligned}
\sup _{h \in \mathcal{H}_{x}^{t+1}\left(\omega^{t}\right) \cap \mathbb{Q}^{d}} & \inf \\
& \text { if } x \geq 0, \\
-\infty & \text { if } x<0,
\end{aligned}\right.
$$

$$
U_{t}\left(\omega^{t}, x\right):=\operatorname{Cl}\left(\mathcal{U}_{t}\right)\left(\omega^{t}, x\right)
$$

Since $\mathcal{U}_{T}$ is u.s.c. [recall Lemma 3.2], it is clear that $U_{T}=\mathcal{U}_{T}$. As already mentioned for $t=0$, we drop the dependency in $\omega_{0}$ and note $U_{0}(x)=U_{0}\left(\omega^{0}, x\right)$. The convention $\infty-\infty=\infty$ is used in the integral in (18) [recall Remark 1.10], where the intersection with $\mathbb{Q}^{d}$ is taken since measurability issues are better handled in this way; see the discussion before Nutz (2016), Lemma 3.6. We introduce the function $I_{t}: \Omega^{t} \times \mathbb{R} \rightarrow[0, \infty]$ which allows us to remove the boundedness assumption of Nutz (2016) and will be used for integrability issues. We set $I_{T}:=U_{T}^{+}$, then for all $0 \leq t \leq T-1, x \in \mathbb{R}$ and $\omega^{t} \in \Omega^{t}$

(20) $I_{t}\left(\omega^{t}, x\right):=1_{[0, \infty)}(x) \sup _{h \in \mathbb{R}^{d}, P \in \mathcal{Q}_{t+1}\left(\omega^{t}\right)} 1_{H_{x}^{t+1}\left(\omega^{t}, P\right)}(h) \int_{\Omega_{t+1}} I_{t+1}\left(\omega^{t}, \cdot, x+1+h \Delta S_{t+1}\left(\omega^{t}, \cdot\right)\right) d P$.

LEMMA 3.26. Assume that Assumptions 1.1 and 1.2 hold true. Let $0 \leq t \leq$ $T-1$ be fixed, $G$ be a fixed nonnegative, real-valued, $\mathcal{B}_{c}\left(\Omega^{t}\right)$-measurable random variable and consider the following random sets $\mathcal{H}^{t+1}:\left(\omega^{t}, x\right) \rightarrow \mathcal{H}_{x}^{t+1}\left(\omega^{t}\right)$ and $\mathcal{D}_{G}^{t+1}: \omega^{t} \rightarrow \mathcal{D}_{G\left(\omega^{t}\right)}^{t+1}\left(\omega^{t}\right)$. They are closed valued, $\operatorname{Graph}\left(\mathcal{H}^{t+1}\right) \in \mathcal{C} A\left(\Omega^{t} \times\right.$ $\left.\mathbb{R} \times \mathbb{R}^{d}\right)$ and $\operatorname{Graph}\left(\mathcal{D}_{G}^{t+1}\right) \in \mathcal{B}_{c}\left(\Omega^{t}\right) \otimes \mathcal{B}\left(\mathbb{R}^{d}\right)$. Moreover, $\left(\omega^{t}, P, h, x\right) \rightarrow$ $1_{H_{x}^{t+1}\left(\omega^{t}, P\right)}(h)$ is $\mathcal{B}\left(\Omega^{t}\right) \otimes \mathcal{B}\left(\mathfrak{P}\left(\Omega_{t+1}\right)\right) \otimes \mathcal{B}\left(\mathbb{R}^{d}\right) \otimes \mathcal{B}(\mathbb{R})$-measurable.

PROOF. It is clear that $\mathcal{H}^{t+1}$ and $\mathcal{D}_{G}^{t+1}$ are closed valued. Lemma A.37 will be in force. First, it allows to prove the last assertion since $\left\{\left(\omega^{t}, P, h, x\right)\right.$, 
$\left.P\left(x+h \Delta S_{t+1}\left(\omega^{t}, \cdot\right) \geq 0\right)=1\right\} \in \mathcal{B}\left(\Omega^{t}\right) \otimes \mathcal{B}\left(\mathfrak{P}\left(\Omega_{t+1}\right)\right) \otimes \mathcal{B}\left(\mathbb{R}^{d}\right) \otimes \mathcal{B}(\mathbb{R})$. Then it shows that

$$
\begin{aligned}
\operatorname{Graph}\left(\mathcal{H}^{t+1}\right) & =\left\{\left(\omega^{t}, x, h\right), \inf _{P \in \mathcal{Q}_{t+1}\left(\omega^{t}\right)} P\left(x+h \Delta S_{t+1}\left(\omega^{t}, \cdot\right) \geq 0\right)=1\right\} \\
& \in \mathcal{C} A\left(\Omega^{t} \times \mathbb{R} \times \mathbb{R}^{d}\right) .
\end{aligned}
$$

Fix some $x \in \mathbb{R}$. For any integer $k \geq 1, r \in \mathbb{Q}, r>0$, we introduce the following $\mathbb{R}^{d}$-valued random variable and random set $\Delta S_{k, t+1}(\cdot):=\Delta S_{t+1}(\cdot) \times$ $1_{\left\{\left|\Delta S_{t+1}(\cdot)\right| \leq k\right\}}(\cdot)$ and $\mathcal{H}_{k, x}^{r, t+1}\left(\omega^{t}\right):=\left\{h \in \mathbb{R}^{d}, x+\Delta S_{k, t+1}\left(\omega^{t}, \cdot\right) \geq r \mathcal{Q}_{t+1}\left(\omega^{t}\right)\right.$-q.s. $\}$ for all $\omega^{t} \in \Omega^{t}$. In the sequel, we will write $\mathcal{H}_{k, x}^{t+1}\left(\omega^{t}\right)$ instead of $\mathcal{H}_{k, x}^{0, t+1}\left(\omega^{t}\right)$. We first prove that $\operatorname{Graph}\left(\mathcal{H}_{x}^{t+1}\right) \in \mathcal{B}_{c}\left(\Omega^{t}\right) \otimes \mathcal{B}\left(\mathbb{R}^{d}\right)$ [recall (16)]. Since $\mathcal{H}_{x}^{t+1}(\cdot)=$ $\bigcap_{k \in \mathbb{N}, k \geq 1} \mathcal{H}_{k, x}^{t+1}(\cdot)$, it is enough to prove that $\operatorname{Graph}\left(\operatorname{Ri}\left(\mathcal{H}_{k, x}^{t+1}\right)\right) \in \mathcal{B}_{c}\left(\Omega^{t}\right) \otimes \mathcal{B}\left(\mathbb{R}^{d}\right)$ for any fixed $k \geq 1$. Indeed from Lemma A.31, for all $\omega^{t} \in \Omega^{t}, \overline{\operatorname{Ri}\left(\mathcal{H}_{k, x}^{t+1}\right)\left(\omega^{t}\right)}=$ $\mathcal{H}_{k, x}^{t+1}\left(\omega^{t}\right)$ and Lemma A.38(i) applies. Since $\Delta S_{k, t+1}$ is bounded, we also get for all $\omega^{t} \in \Omega^{t}$ that $\operatorname{Ri}\left(\mathcal{H}_{k, x}^{t+1}\right)\left(\omega^{t}\right)=\bigcup_{r \in \mathbb{Q}, r>0} \mathcal{H}_{k, x}^{r, t+1}\left(\omega^{t}\right)$. Using Lemmata A.37 and A.36, we obtain that for all $r \in \mathbb{Q}, r>0, \operatorname{Graph}\left(\mathcal{H}_{k, x}^{r, t+1}\right)$ and also $\operatorname{Graph}\left(\operatorname{Ri}\left(\mathcal{H}_{k, x}^{t+1}\right)\right)$ are co-analytic sets. Lemma A.38(ii) implies that $\operatorname{Graph}\left(\operatorname{Ri}\left(\mathcal{H}_{k, x}^{t+1}\right)\right) \in \mathcal{B}_{c}\left(\Omega^{t}\right) \otimes \mathcal{B}\left(\mathbb{R}^{d}\right)$.

Now let $\mathcal{H}_{G}^{t+1}: \omega^{t} \rightarrow \mathcal{H}_{G\left(\omega^{t}\right)}^{t+1}\left(\omega^{t}\right)$ then it is easy to see that

$$
\begin{aligned}
\operatorname{Graph}\left(\mathcal{H}_{G}^{t+1}\right)= & \bigcap_{n \in \mathbb{N}, n \geq 1} \bigcup_{q \in \mathbb{Q}, q \geq 0}\left\{\left(\omega^{t}, h\right) \in \Omega^{t} \times \mathbb{R} \times \mathbb{R}^{d},\right. \\
& \left.q \leq G\left(\omega^{t}\right) \leq q+\frac{1}{n}, h \in \operatorname{Graph}\left(\mathcal{H}_{q+\frac{1}{n}}^{t+1}\right)\right\} \\
\in & \mathcal{B}_{c}\left(\Omega^{t}\right) \otimes \mathcal{B}\left(\mathbb{R}^{d}\right),
\end{aligned}
$$

since $G$ is $\mathcal{B}_{c}\left(\Omega^{t}\right)$-measurable. So using Lemma 2.2 and that $\operatorname{Graph}\left(\mathcal{D}_{G}^{t+1}\right)=$ $\operatorname{Graph}\left(\mathcal{H}_{G}^{t+1}\right) \cap \operatorname{Graph}\left(D^{t+1}\right)$, we obtain that $\operatorname{Graph}\left(\mathcal{D}_{G}^{t+1}\right) \in \mathcal{B}_{c}\left(\Omega^{t}\right) \otimes \mathcal{B}\left(\mathbb{R}^{d}\right)$, which concludes the proof.

We introduce for all $r \in \mathbb{Q}, r>0$

$$
\begin{aligned}
& J_{T}^{r}\left(\omega^{T}\right):=U_{T}^{-}\left(\omega^{T}, r\right) \quad \text { for } \omega^{T} \in \Omega^{T} \\
& J_{t}^{r}\left(\omega^{t}\right):=\sup _{P \in \mathcal{Q}_{t+1}\left(\omega^{t}\right)} \int_{\Omega_{t+1}} J_{t+1}^{r}\left(\omega^{t}, \cdot\right) d P \quad \text { for } t \in\{0, \ldots, T-1\}, \omega^{t} \in \Omega^{t} .
\end{aligned}
$$

As usual, we will write $J_{0}^{r}=J_{0}^{t}\left(\omega^{0}\right)$. 
Proposition 3.27. Assume that Assumptions 1.1 and 3.1 hold true. Then for any $t \in\{0, \ldots, T\}, r \in \mathbb{Q}, r>0$, the function $\omega^{t} \rightarrow J_{t}^{r}\left(\omega^{t}\right)$ is well defined, nonnegative, u.s.a. and verifies $\sup _{P \in \mathcal{Q}^{t}} E_{P} J_{t}^{r}<\infty$. Furthermore, there exists some $\mathcal{Q}^{t}$-full measure set $\widehat{\Omega}^{t} \in \mathcal{C} A\left(\Omega^{t}\right)$ on which $J_{t}^{r}(\cdot)<\infty$.

PROOF. We proceed by induction on $t$. Fix some $r \in \mathbb{Q}, r>0$. For $t=T$, $J_{T}^{r}(\cdot)=U_{T}^{-}(\cdot, r)$ is nonnegative and u.s.a. [see Definition 1.6, Lemma 3.2 and (1)]. We have that $\sup _{P \in \mathcal{Q}^{T}} E_{P}\left(J_{T}^{r}\right)<\infty$ by Assumption 3.1. Using Lemma 3.2, $\widehat{\Omega}^{T}:=\Omega_{\text {Dom }}^{T} \in \mathcal{B}\left(\Omega^{T}\right) \subset \mathcal{C} A\left(\Omega^{T}\right)$ [see (1)], $P\left(\widehat{\Omega}^{T}\right)=1$ for all $P \in \mathcal{Q}^{T}$ and $J_{T}^{r}<\infty$ on $\widehat{\Omega}^{T}$. Assume now that for some $t \leq T-1, J_{t+1}^{r}$ is nonnegative, u.s.a. and that $\sup _{P \in \mathcal{Q}^{t+1}} E_{P}\left(J_{t+1}^{r}\right)<\infty$. As $J_{t+1}^{r}(\cdot) \geq 0$, it is clear that $J_{t}^{r}(\cdot) \geq 0$ holds true. We now apply Bertsekas and Shreve (2004), Proposition 7.48, page $180,{ }^{3}$ with $X=\Omega^{t} \times \mathfrak{P}\left(\Omega_{t+1}\right), Y=\Omega_{t+1}, f\left(\omega^{t}, P, \omega_{t+1}\right)=J_{t+1}^{r}\left(\omega^{t}, \omega_{t+1}\right)$ and $q\left(d \omega_{t+1} \mid \omega^{t}, P\right)=P\left(d \omega_{t+1}\right)$. Indeed $f$ is u.s.a. [see Bertsekas and Shreve (2004), Proposition 7.38, page 165], $\left(\omega^{t}, P\right) \rightarrow P\left(d \omega_{t+1}\right) \in \mathfrak{P}\left(\Omega_{t+1}\right)$ is a $\mathcal{B}\left(\Omega^{t}\right) \otimes \mathcal{B}\left(\mathfrak{P}\left(\Omega_{t+1}\right)\right)$-measurable stochastic kernel. So we get that $j_{t}^{r}:\left(\omega^{t}, P\right) \rightarrow$ $\int_{\Omega_{t+1}} J_{t+1}^{r}\left(\omega^{t}, \omega_{t+1}\right) P\left(d \omega_{t+1}\right)$ is u.s.a. As Assumption 1.1 holds true [recall that $\operatorname{Proj}_{\Omega^{t}}\left(\operatorname{Graph}\left(\mathcal{Q}_{t+1}\right)\right)=\Omega^{t}$ ], Bertsekas and Shreve (2004), Proposition 7.47, page 179, applies and $\omega^{t} \rightarrow \sup _{P \in \mathcal{Q}_{t+1}\left(\omega^{t}\right)} j_{t}^{r}\left(\omega^{t}, P\right)=J_{t}^{r}\left(\omega^{t}\right)$ is u.s.a. We set $\Omega_{r}^{t}:=\left\{\omega^{t} \in \Omega^{t}, J_{t}^{r}\left(\omega^{t}\right)<\infty\right\}$, then $\Omega_{r}^{t}=\bigcup_{n \geq 1}\left\{\omega^{t} \in \Omega^{t}, J_{t}^{r}\left(\omega^{t}\right) \leq n\right\} \in \mathcal{C} A\left(\Omega^{t}\right)$. Fix some $\varepsilon>0$. From Bertsekas and Shreve (2004), Proposition 7.50, page 184 [recall Assumption 1.1], there exists some analytically measurable $p_{\varepsilon}: \omega^{t} \rightarrow$ $\mathfrak{P}\left(\Omega_{t+1}\right)\left[p_{\varepsilon} \in \mathcal{S} K_{t+1}\right]$, such that $p_{\varepsilon}\left(\cdot, \omega^{t}\right) \in \mathcal{Q}_{t+1}\left(\omega^{t}\right)$ for all $\omega^{t} \in \Omega^{t}$ and

$$
\begin{aligned}
j_{t}^{r}\left(\omega^{t}, p_{\varepsilon}\right) & =\int_{\Omega_{t+1}} J_{t+1}^{r}\left(\omega^{t}, \omega_{t+1}\right) p_{\varepsilon}\left(d \omega_{t+1}, \omega^{t}\right) \\
& \geq \begin{cases}J_{t}^{r}\left(\omega^{t}\right)-\varepsilon & \text { if } \omega^{t} \in \Omega_{r}^{t}, \\
\frac{1}{\varepsilon} & \text { otherwise. }\end{cases}
\end{aligned}
$$

Assume that $\Omega_{r}^{t}$ is not a $\mathcal{Q}^{t}$-full measure set. Then there exists some $P^{*} \in \mathcal{Q}^{t}$ such that $P^{*}\left(\Omega_{r}^{t}\right)<1$. Set $P_{\varepsilon}^{*}:=P^{*} \otimes p_{\varepsilon}$ then $P_{\varepsilon}^{*} \in \mathcal{Q}^{t+1}$ [see (2)] and we have that

$$
\sup _{P \in \mathcal{Q}^{t+1}} E_{P} J_{t+1}^{r} \geq E_{P_{\varepsilon}^{*}} J_{t+1}^{r} \geq \frac{1}{\varepsilon}\left(1-P^{*}\left(\Omega_{r}^{t}\right)\right)-\varepsilon P^{*}\left(\Omega_{r}^{t}\right) .
$$

As the previous inequality holds true for all $\varepsilon>0$, letting $\varepsilon$ go to 0 we obtain that $\sup _{P \in \mathcal{Q}^{t+1}} E_{P}\left(J_{t+1}^{r}\right)=+\infty$ : a contradiction and $\Omega_{r}^{t}$ is a $\mathcal{Q}^{t}$-full measure set. Now, for all $P \in \mathcal{Q}^{t}$, we set $P_{\varepsilon}=P \otimes p_{\varepsilon} \in \mathcal{Q}^{t+1}$ [see (2)]. Then, using (23) we get that

$$
E_{P} J_{t}^{r}-\varepsilon=E_{P} 1_{\Omega_{r}^{t}} J_{t}^{r}-\varepsilon \leq E_{P_{\varepsilon}} J_{t+1}^{r} \leq \sup _{P \in \mathcal{Q}^{t+1}} E_{P}\left(J_{t+1}^{r}\right) \text {. }
$$

\footnotetext{
${ }^{3}$ As we will often use similar arguments in the rest of the paper, we provide some details at this stage.
} 
Again, as this is true for all $\varepsilon>0$ and all $P \in \mathcal{Q}^{t}$ we obtain that $\sup _{P \in \mathcal{Q}^{t}} E_{P}\left(J_{t}^{r}\right) \leq$ $\sup _{P \in \mathcal{Q}^{t+1}} E_{P}\left(J_{t+1}^{r}\right)<\infty$. Finally, we set $\widehat{\Omega}^{t}=\bigcap_{r \in \mathbb{Q}, r>0} \Omega_{r}^{t}$. It is clear that $\widehat{\Omega}^{t} \in$ $\mathcal{C} A\left(\Omega^{t}\right)$ is a $\mathcal{Q}^{t}$-full measure set and that $J_{t}^{r}(\cdot)<\infty$ on $\widehat{\Omega}^{t}$ for all $r \in \mathbb{Q}, r>0$.

Let $1 \leq t \leq T$ be fixed. We introduce the following notation: for any $\mathcal{B}_{c}\left(\Omega^{t-1}\right)$ measurable random variable $G$ and any $P \in \mathcal{Q}^{t}, \phi_{t}(G, P)$ is the set of all $\mathcal{B}_{c}\left(\Omega^{t-1}\right)$-measurable random variable $\xi$ (one-step strategy), such that $G(\cdot)+$ $\xi \Delta S_{t}(\cdot) \geq 0 P$-a.s. Propositions 3.28 to 3.30 solve the dynamic programming procedure and hold true under the following set of conditions.

$$
\begin{aligned}
& \forall \omega^{t} \in \Omega^{t}, U_{t}\left(\omega^{t}, \cdot\right): \mathbb{R} \rightarrow \mathbb{R} \cup\{ \pm \infty\} \text { is nondecreasing, u.s.c. and } \\
& \text { concave on } \mathbb{R} \text {, } \\
& \forall \omega^{t} \in \Omega^{t}, I_{t}\left(\omega^{t}, \cdot\right): \mathbb{R} \rightarrow \mathbb{R} \cup\{+\infty\} \text { is nondecreasing and } \\
& \text { nonnegative on } \mathbb{R} \text {, } \\
& U_{t} \in \mathcal{L} S A\left(\Omega^{t} \times \mathbb{R}\right), \\
& I_{t} \in \mathcal{U} S A\left(\Omega^{t} \times \mathbb{R}\right) \text {, } \\
& U_{t}\left(\omega^{t}, x\right) \leq I_{t}\left(\omega^{t}, x+1\right) \quad \text { for all }\left(\omega^{t}, x\right) \in \Omega^{t} \times \mathbb{R}, \\
& \sup _{P \in \mathcal{Q}^{t}} \sup _{\xi \in \phi_{t}(G, P)} \int_{\Omega^{t}} I_{t}\left(\omega^{t}, G\left(\omega^{t-1}\right)+\xi\left(\omega^{t-1}\right) \Delta S_{t}\left(\omega^{t}\right)\right) P\left(d \omega^{t}\right)<\infty \text {, }
\end{aligned}
$$

for any $G:=x+\sum_{s=1}^{t-1} \phi_{s} \Delta S_{s}$, where $x \geq 0,\left(\phi_{s}\right)_{1 \leq s \leq t-1}$ is universally predictable,

$$
U_{t}\left(\omega^{t}, r\right) \geq-J_{t}^{r}\left(\omega^{t}\right) \quad \text { for all } \omega^{t} \in \Omega^{t}, \text { all } r \in \mathbb{Q}, r>0
$$

Proposition 3.28. Let $0 \leq t \leq T-1$ be fixed. Assume that the $N A\left(\mathcal{Q}^{T}\right)$ condition, that Assumptions 1.1, 1.2, 1.4 hold true and that (24), (25), (26), (27), (28), (29) and (30) hold true at stage $t+1$. Then there exists some $\mathcal{Q}^{t}$-full measure set $\widetilde{\Omega}^{t} \in \mathcal{B}_{c}\left(\Omega^{t}\right)$ such that for all $\omega^{t} \in \widetilde{\Omega}^{t}$ the function $\left(\omega_{t+1}, x\right) \rightarrow U_{t+1}\left(\omega^{t}, \omega_{t+1}, x\right)$ satisfies the assumptions of Theorem 3.23 (or Lemmata 3.21 and 3.22) with $\bar{\Omega}=$ $\Omega_{t+1}, \mathcal{G}=\mathcal{B}_{c}\left(\Omega_{t+1}\right), \mathcal{Q}=\mathcal{Q}_{t+1}\left(\omega^{t}\right), Y(\cdot)=\Delta S_{t+1}\left(\omega^{t}, \cdot\right), V(\cdot, \cdot)=U_{t+1}\left(\omega^{t}, \cdot, \cdot\right)$ where $V$ is defined on $\Omega_{t+1} \times \mathbb{R}$ (shortly called context $t+1$ from now on).

Note that under the assumptions of Proposition 3.28, for all $\omega^{t} \in \widetilde{\Omega}^{t}$ and $x \geq 0$ we have that [see (14), (18) and (19)]

$$
U_{t}\left(\omega^{t}, x\right)=\mathcal{U}_{t}\left(\omega^{t}, x\right)=\sup _{h \in \mathcal{H}_{x}^{t+1}\left(\omega^{t}\right)} \inf _{P \in \mathcal{Q}_{t+1}\left(\omega^{t}\right)} \int_{\Omega_{t+1}} U_{t+1}\left(\omega^{t}, \cdot, x+h \Delta S_{t+1}\left(\omega^{t}, \cdot\right)\right) d P
$$

PROOF. To prove the proposition, we will review one by one the assumptions needed to apply Theorem 3.23 in the context $t+1$. First, from Assumption 1.4 
for $\omega^{t} \in \Omega^{t}$ fixed, we have that $Y_{i}(\cdot)=\Delta S_{t+1}^{i}\left(\omega^{t}, \cdot\right) \geq-b:=-\max (1+s+$ $\left.S_{t}^{i}\left(\omega^{t}\right), i \in\{1, \ldots, d\}\right)$ and $0<b<\infty$ : Assumption 3.7 holds true. From (24) at $t+1$ for all $\omega^{t} \in \Omega^{t}$ and $\omega_{t+1} \in \Omega_{t+1}, U_{t+1}\left(\omega^{t}, \omega_{t+1}, \cdot\right)$ is nondecreasing, u.s.c. and concave on $\mathbb{R}$. From (26) at $t+1, U_{t+1}$ is $\mathcal{B}_{c}\left(\Omega^{t+1} \times \mathbb{R}\right)$-measurable. Fix some $x \in \mathbb{R}$ and $\omega^{t} \in \Omega^{t}$, then $\omega_{t+1} \rightarrow U_{t+1}\left(\omega^{t}, \omega_{t+1}, x\right)$ is $\mathcal{B}_{c}\left(\Omega_{t+1}\right)$-measurable; see Bertsekas and Shreve (2004), Lemma 7.29, page 174. Thus Assumption 3.12 is satisfied in the context $t+1$.

We now prove the assumptions that are verified for $\omega^{t}$ in some well-chosen $\mathcal{Q}^{t}$ full measure set. First, from Proposition 2.3, for all $\omega^{t} \in \Omega_{N A}^{t}$, Assumptions 3.8 and 3.9 hold true in the context $t+1$. Fix $\omega^{t} \in \widehat{\Omega}^{t}$ and some $r \in \mathbb{Q}, r>0$. Using (30) at $t+1$ and Proposition 3.27, we get that

$$
\begin{aligned}
& \sup _{P \in \mathcal{Q}_{t+1}\left(\omega^{t}\right)} \int_{\Omega_{t+1}} U_{t+1}^{-}\left(\omega^{t}, \omega_{t+1}, r\right) P\left(d \omega^{t}\right) \\
& \quad \leq \sup _{P \in \mathcal{Q}_{t+1}\left(\omega^{t}\right)} \int_{\Omega_{t+1}} J_{t+1}^{r}\left(\omega^{t}, \omega_{t+1}\right) P\left(d \omega^{t}\right)=J_{t}^{r}\left(\omega^{t}\right)<\infty,
\end{aligned}
$$

and Assumption 3.13 in context $t+1$ is verified for all $\omega^{t} \in \widehat{\Omega}^{t}$. We finish with Assumption 3.16 in context $t+1$ whose proof is more involved. We want to show that for $\omega^{t}$ in some $\mathcal{Q}^{t}$-full measure set to be determined, for all $h \in \mathcal{H}_{1}^{t+1}\left(\omega^{t}\right)$ and $P \in \mathcal{Q}_{t+1}\left(\omega^{t}\right)$ we have that

$$
\int_{\Omega_{t+1}} U_{t+1}^{+}\left(\omega^{t}, \cdot, 1+h \Delta S_{t+1}\left(\omega^{t}, \cdot\right)\right) d P<\infty .
$$

Let $i_{t}\left(\omega^{t}, h, P\right)=\int_{\Omega_{t+1}} I_{t+1}\left(\omega^{t}, \omega_{t+1}, 2+h \Delta S_{t+1}\left(\omega^{t}, \omega_{t+1}\right)\right) P\left(d \omega_{t+1}\right)$ and $I^{t}\left(\omega^{t}\right):=\left\{(h, P) \in \mathbb{R}^{d} \times \mathcal{Q}_{t+1}\left(\omega^{t}\right), P\left(1+h \Delta S_{t+1}\left(\omega^{t}, \cdot\right) \geq 0\right)=1, i_{t}\left(\omega^{t}, h, P\right)=\right.$ $\infty\}$. Fix some $\omega^{t} \in \Omega^{t}$, then using (25) and (28) at $t+1$ we have that if $h \in \mathcal{H}_{1}^{t+1}\left(\omega^{t}\right)$ and $P \in \mathcal{Q}_{t+1}\left(\omega^{t}\right)$ are such that (32) does not hold true, then $(h, P) \in I^{t}\left(\omega^{t}\right)$. Thus (32) holds true for all $h \in \mathcal{H}_{1}^{t+1}\left(\omega^{t}\right)$ and $P \in \mathcal{Q}_{t+1}\left(\omega^{t}\right)$ if $\omega^{t} \in\left\{I^{t}=\varnothing\right\}$ and if this set is of $\mathcal{Q}^{t}$-full measure, Assumption 3.16 in context $t+1$ is proved. We first prove that $\operatorname{Graph}\left(I^{t}\right) \in \mathcal{A}\left(\Omega^{t} \times \mathbb{R}^{d} \times \mathfrak{P}\left(\Omega_{t+1}\right)\right)$. From (27) at $t+1$, Assumption 1.2 and Bertsekas and Shreve (2004), Lemma 7.30(3), page 178, $\left(\omega^{t}, h, \omega_{t+1}\right) \rightarrow I_{t+1}\left(\omega^{t}, \omega_{t+1}, 2+h \Delta S_{t+1}\left(\omega^{t}, \omega_{t+1}\right)\right)$ is u.s.a. Then using Bertsekas and Shreve (2004), Proposition 7.48, page 180 [which can be used with similar arguments as in the proof of Proposition 3.27], we get that $i_{t}$ is u.s.a. It follows that

$$
i_{t}^{-1}(\{\infty\})=\bigcap_{n \geq 1}\left\{\left(\omega^{t}, h, P\right), i_{t}\left(\omega^{t}, h, P\right)>n\right\} \in \mathcal{A}\left(\Omega^{t} \times \mathbb{R}^{d} \times \mathfrak{P}\left(\Omega_{t+1}\right)\right) .
$$

Now using Assumption 1.1 together with Lemma A.37 we get that $\left\{\left(\omega^{t}, h, P\right)\right.$, $\left.P \in \mathcal{Q}_{t+1}\left(\omega^{t}\right), P\left(1+h \Delta S_{t+1}\left(\omega^{t}, \cdot\right) \geq 0\right)=1\right\} \in \mathcal{A}\left(\Omega^{t} \times \mathbb{R}^{d} \times \mathfrak{P}\left(\Omega_{t+1}\right)\right)$ and the fact that $\operatorname{Graph}\left(I^{t}\right)$ and $\operatorname{Proj}_{\Omega^{t}}\left(\operatorname{Graph}\left(I^{t}\right)\right)=\left\{I^{t} \neq \varnothing\right\}$ are analytic sets 
follows immediately [recall Bertsekas and Shreve (2004), Proposition 7.39, page 165]. Applying the Jankov-von Neumann projection theorem [see Bertsekas and Shreve (2004), Proposition 7.49, page 182], we obtain that there exists some analytically measurable and, therefore, $\mathcal{B}_{c}\left(\Omega^{t}\right)$-measurable function $\omega^{t} \in$ $\left\{I^{t} \neq \varnothing\right\} \rightarrow\left(h^{*}\left(\omega^{t}\right), p^{*}\left(\cdot, \omega^{t}\right)\right) \in \mathbb{R}^{d} \times \mathfrak{P}\left(\Omega_{t+1}\right)$ such that for all $\omega^{t} \in\left\{I^{t} \neq \varnothing\right\}$, $\left(h^{*}\left(\omega^{t}\right), p^{*}\left(\cdot, \omega^{t}\right)\right) \in I^{t}\left(\omega^{t}\right)$. We may and will extend $h^{*}$ and $p^{*}$ on all $\Omega^{t}$ so that $h^{*}$ and $p^{*}$ remain $\mathcal{B}_{c}\left(\Omega^{t}\right)$-measurable.

We prove now by contradiction that $\left\{I^{t}=\varnothing\right\}$ is a $\mathcal{Q}^{t}$-full measure set. Assume that there exists some $\widetilde{P} \in \mathcal{Q}^{t}$ such that $\widetilde{P}\left(\left\{I^{t} \neq \varnothing\right\}\right)>0$ and set $\widetilde{P}^{*}=\widetilde{P} \otimes p^{*}$. Since $p^{*} \in S K_{t+1}$ and $p^{*}\left(\cdot, \omega^{t}\right) \in \mathcal{Q}_{t+1}\left(\omega^{t}\right)$ for all $\omega^{t} \in \Omega^{t}, \widetilde{P}^{*} \in \mathcal{Q}^{t+1}$ [see (2)]. It is also clear that $\widetilde{P}^{*}\left(2+h^{*}(\cdot) \Delta S_{t+1}(\cdot) \geq 0\right)=1$. Now for all $\omega^{t} \in\left\{I^{t} \neq \varnothing\right\}$, we have that $i_{t}\left(\omega^{t}, h^{*}\left(\omega^{t}\right), p^{*}\left(\cdot, \omega^{t}\right)\right)=\infty$, and thus

$\int_{\Omega^{t+1}} I_{t+1}\left(\omega^{t+1}, 2+h^{*}\left(\omega^{t}\right) \Delta S_{t+1}\left(\omega^{t+1}\right)\right) \widetilde{P}^{*}\left(d \omega^{t+1}\right) \geq \int_{\left\{I^{t} \neq \varnothing\right\}}(+\infty) \widetilde{P}\left(d \omega^{t}\right)=+\infty$

a contradiction with (29) at $t+1$.

We can now define $\widetilde{\Omega}^{t}:=\left\{I^{t}=\varnothing\right\} \cap \widehat{\Omega}^{t} \cap \Omega_{N A}^{t} \subset \widehat{\Omega}^{t}$. It is clear, recalling Propositions 2.3 and 3.27, that $\widetilde{\Omega}^{t} \in \mathcal{B}_{c}\left(\Omega^{t}\right)$ is a $\mathcal{Q}^{t}$-full measure set and the proof is complete.

The next proposition enables us to initialize the induction procedure that will be carried on in the proof of the main theorem.

Proposition 3.29. Assume that the $N A\left(\mathcal{Q}^{T}\right)$ condition, Assumptions 3.1 and 3.5 hold true. Then (24), (25), (26), (27), (28), (29) and (30) hold true for $t=T$.

Proof. As $U_{T}=U 1_{\Omega_{\mathrm{Dom}}^{T} \times[0, \infty) \cup \Omega^{T} \times(-\infty, 0)}$ and $I_{T}=U_{T}^{+}$, using Definition 1.6, (25), (28) and (30) [recall (21)] for $t=T$ are true. For all $\omega^{T} \in \Omega^{T}$, $U_{T}\left(\omega^{T}, \cdot\right)$ is also right-continuous and u.s.c. [see Lemma 3.2], thus (24) also holds true. Moreover, $U_{T}(\cdot, x)$ is $\mathcal{B}\left(\Omega^{T}\right)$-measurable for all $x \in \mathbb{R}$, thus $U_{T}$ is $\mathcal{B}\left(\Omega^{T}\right) \otimes \mathcal{B}(\mathbb{R})$-measurable [see Blanchard, Carassus and Rásonyi (2016), Lemma 7.16] and (26) and (27) hold true for $t=T$. It remains to prove that (29) is true for $t=T$. Let $G:=x+\sum_{t=1}^{T-1} \phi_{t} \Delta S_{t}$ where $x \geq 0$ and $\left(\phi_{s}\right)_{1 \leq s \leq T-1}$ is universally predictable. Fix some $P \in \mathcal{Q}^{T}$ and $\xi \in \phi_{T}(G, P)$. Let $\left(\phi_{i}^{\xi}\right)_{1 \leq i \leq T} \in$ $\Phi$ be defined by $\phi_{T}^{\xi}=\xi$ and $\phi_{s}^{\xi}=\phi_{s}$ for $1 \leq s \leq T-1$, then $V_{T}^{x, \phi^{\xi}}=$ $G+\xi \Delta S_{T}, \phi^{\xi} \in \Phi(x, P), \int_{\Omega^{T}} I_{T}\left(\omega^{T}, G\left(\omega^{T-1}\right)+\xi\left(\omega^{T-1}\right) \Delta S_{T}\left(\omega^{T}\right)\right) P\left(d \omega^{T}\right)=$ $E_{P} U^{+}\left(\cdot, V_{T}^{x, \phi^{\xi}}(\cdot)\right)$ and (29) follows from Proposition 3.25.

The next proposition proves the induction step. 
Proposition 3.30. Let $0 \leq t \leq T-1$ be fixed. Assume that the $N A\left(\mathcal{Q}^{T}\right)$ condition holds true as well as Assumptions 1.1, 1.2, 1.4 and (24), (25), (26), (27), (28), (29) and (30) at $t+1$. Then (24), (25), (26), (27), (28), (29) and (30) are true for $t$.

Moreover, for all $X=x+\sum_{s=1}^{t} \phi_{s} \Delta S_{s}$, where $x \geq 0,\left(\phi_{s}\right)_{1 \leq s \leq t}$ is universally predictable and $\{X \geq 0\}$ is $\mathcal{Q}^{t}$-full measure set, there exists some $\mathcal{Q}^{t}$-full measure set $\Omega_{X}^{t} \in \mathcal{B}_{c}\left(\Omega^{t}\right)$, such that $\Omega_{X}^{t} \subset \widetilde{\Omega}^{t}$ (see Proposition 3.28 for the definition of $\left.\widetilde{\Omega}^{t}\right)$ and some $\mathcal{B}_{c}\left(\Omega^{t}\right)$-measurable random variable $\widehat{h}_{t+1}^{X}$ such that for all $\omega^{t} \in \Omega_{X}^{t}$, $\widehat{h}_{t+1}^{X}\left(\omega^{t}\right) \in \mathcal{D}_{X\left(\omega^{t}\right)}^{t+1}\left(\omega^{t}\right)$ and

$$
U_{t}\left(\omega^{t}, X\left(\omega^{t}\right)\right)=\inf _{P \in \mathcal{Q}_{t+1}\left(\omega^{t}\right)} \int_{\Omega_{t+1}} U_{t+1}\left(\omega^{t}, \cdot, X\left(\omega^{t}\right)+\widehat{h}_{t+1}^{X}\left(\omega^{t}\right) \Delta S_{t+1}\left(\omega^{t}, \cdot\right)\right) d P
$$

ProOF. First, we prove that (24) is true at $t$. We fix some $\omega^{t} \in \Omega^{t}$. From (24) at $t+1$, the function $U_{t+1}\left(\omega^{t}, \omega_{t+1}, \cdot\right)$ is u.s.c., concave and nondecreasing on $\mathbb{R}$ for all $\omega_{t+1} \in \Omega_{t+1}$. From (18) and (19), $U_{t+1}\left(\omega^{t}, \omega_{t+1}, x\right)=-\infty$ for all $x<0$ and $\omega_{t+1} \in \Omega_{t+1}$. Then using (26) at $t+1$ and Lemma A.36, we find that $U_{t+1}\left(\omega^{t}, \cdot, x\right)$ is $\mathcal{B}_{c}\left(\Omega_{t+1}\right)$-measurable for all $x \in \mathbb{R}$. Hence, Assumption 3.12 of Lemma 3.18 holds true in the context $t+1$ and we obtain that $x \rightarrow U_{t}\left(\omega^{t}, x\right)=$ $\mathrm{Cl}\left(\mathcal{U}_{t}\right)\left(\omega^{t}, x\right)$ [see (18) and (19)] is u.s.c., concave and nondecreasing. As this is true for all $\omega^{t} \in \Omega^{t},(24)$ at $t$ is proved. Note that we also obtain that $x \rightarrow \mathcal{U}_{t}\left(\omega^{t}, x\right)$ is nondecreasing for all $\omega^{t} \in \Omega^{t}$. Now we prove (26) at $t$. Since integrals might not always be well defined, we need to be a bit cautious. We introduce first $u_{t}$ and $\widehat{u}_{t}: \Omega^{t} \times \mathbb{R}^{d} \times[0, \infty) \times \mathfrak{P}\left(\Omega_{t+1}\right) \rightarrow \mathbb{R} \cup\{ \pm \infty\}$

$$
\begin{aligned}
& u_{t}\left(\omega^{t}, h, x, P\right)=\int_{\Omega_{t+1}} U_{t+1}\left(\omega^{t}, \omega_{t+1}, x+h \Delta S_{t+1}\left(\omega^{t}, \omega_{t+1}\right)\right) P\left(d \omega_{t+1}\right), \\
& \widehat{u}_{t}\left(\omega^{t}, h, x, P\right)=1_{\mathcal{H}_{x}^{t+1}\left(\omega^{t}\right)}(h) u_{t}\left(\omega^{t}, h, x, P\right)+(-\infty) 1_{\mathbb{R}^{d} \backslash \mathcal{H}_{x}^{t+1}\left(\omega^{t}\right)}(h) .
\end{aligned}
$$

As $U_{t+1}$ is 1.s.a. [see (26) at $t+1$ ] and Assumption 1.2 holds true, Bertsekas and Shreve (2004), Lemma 7.30(3), page 177, implies that $\left(\omega^{t}, \omega_{t+1}, h, x\right) \rightarrow$ $U_{t+1}\left(\omega^{t}, \omega_{t+1}, x+h \Delta S_{t+1}\left(\omega^{t}, \omega_{t+1}\right)\right)$ is 1.s.a. So Bertsekas and Shreve (2004), Proposition 7.48, page 180 [recall the convention $\infty-\infty=\infty$, see $\operatorname{Re}$ mark 1.10], shows that $u_{t}$ is 1.s.a. Fix some $c \in \mathbb{R}$ and set $\widehat{C}:=\widehat{u}_{t}^{-1}((-\infty, c))$, $C:=u_{t}^{-1}((-\infty, c)), A:=\left\{\left(\omega^{t}, h, x\right), h \in \mathcal{H}_{x}^{t+1}\left(\omega^{t}\right)\right\} \times \mathfrak{P}\left(\Omega_{t+1}\right)$ and $A^{c}:=$ $\left\{\left(\omega^{t}, h, x\right), h \notin \mathcal{H}_{x}^{t+1}\left(\omega^{t}\right)\right\} \times \mathfrak{P}\left(\Omega_{t+1}\right)$, then $\widehat{C}=(C \cap A) \cup A^{c}=C \cup A^{c}$. As $u_{t}$ is 1.s.a., $C$ is an analytic set. Lemma 3.26 implies that $A^{c}=\left\{\left(\omega^{t}, h, x\right),\left(\omega^{t}, x, h\right) \notin\right.$ $\left.\operatorname{Graph}\left(\mathcal{H}^{t+1}\right)\right\} \times \mathfrak{P}\left(\Omega_{t+1}\right)$, and thus $\widehat{C}$, are analytic sets and $\widehat{u}_{t}$ is 1.s.a. Using Assumption 1.1 and Bertsekas and Shreve (2004), Proposition 7.47, page 179, we get that

$$
\tilde{u}_{t}:\left(\omega^{t}, h, x\right) \rightarrow \inf _{P \in \mathcal{Q}_{t+1}\left(\omega^{t}\right)} \widehat{u}_{t}\left(\omega^{t}, h, x, P\right) \in \mathcal{L} S A\left(\Omega^{t} \times \mathbb{R}^{d} \times \mathbb{R}\right)
$$


Then Bertsekas and Shreve (2004), Lemma 7.30(2), page 178, implies that $\tilde{\mathcal{U}}_{t}$ : $\left(\omega^{t}, x\right) \rightarrow \sup _{h \in \mathbb{Q}^{d}} \tilde{u}_{t}\left(\omega^{t}, h, x\right)$ is 1.s.a. and since $\tilde{\mathcal{U}}_{t}=\mathcal{U}_{t}$ on $\Omega^{t} \times[0, \infty)$, it follows that $\mathcal{U}_{t}$ is 1. s.a. We have already seen that $\omega^{t} \in \Omega^{t}, \mathcal{U}_{t}\left(\omega^{t}, \cdot\right)$ is nondecreasing thus, for all $\omega^{t} \in \Omega^{t}$ and $x \in \mathbb{R}$ we get that [recall (19)]

$$
U_{t}\left(\omega^{t}, x\right)=\operatorname{Cl}\left(\mathcal{U}_{t}\right)\left(\omega^{t}, x\right)=\limsup _{y \rightarrow x} \mathcal{U}_{t}\left(\omega^{t}, y\right)=\lim _{n \rightarrow \infty} \mathcal{U}_{t}\left(\omega^{t}, x+\frac{1}{n}\right) .
$$

As $\left(\omega^{t}, x\right) \rightarrow \mathcal{U}_{t}\left(\omega^{t}, x+\frac{1}{n}\right)$ is l.s.a., Bertsekas and Shreve (2004), Lemma 7.30(2), page 178, implies that $U_{t}$ is also l.s.a. We prove now that (27) holds true for $t$. We introduce $\hat{\mathbf{i}}_{t}: \Omega^{t} \times \mathbb{R}^{d} \times[0, \infty) \times \mathfrak{P}\left(\Omega_{t+1}\right) \rightarrow \mathbb{R} \cup\{+\infty\}$ [recall (15)],

$$
\hat{\mathbf{i}}_{t}\left(\omega^{t}, h, x, P\right)=1_{H_{x}^{t+1}\left(\omega^{t}, P\right)}(h) \int_{\Omega_{t+1}} I_{t+1}\left(\omega^{t}, \cdot, x+1+h \Delta S_{t+1}\left(\omega^{t}, \cdot\right)\right) d P .
$$

Note that, using (25) at $t+1$, the integral in (35) is well defined (potentially infinite valued). Using Assumption 1.2, (27) at $t+1$ and Bertsekas and Shreve (2004), Lemma 7.30(3), page 177, we find that $\left(\omega^{t+1}, h, x, P\right) \rightarrow I_{t+1}\left(\omega^{t}, \omega_{t+1}, x+1+\right.$ $\left.h \Delta S_{t+1}\left(\omega^{t}, \omega_{t+1}\right)\right)$ is u.s.a. Thus Bertsekas and Shreve (2004), Proposition 7.48, page 180 , applies ${ }^{4}$ and

$$
\begin{aligned}
\left(\omega^{t}, h, x, P\right) & \rightarrow \int_{\Omega_{t+1}} I_{t+1}\left(\omega^{t}, \cdot, x+1+h \Delta S_{t+1}\left(\omega^{t}, \cdot\right)\right) d P \\
& \in \mathcal{U} S A\left(\Omega^{t} \times \mathbb{R}^{d} \times \mathbb{R} \times \mathfrak{P}\left(\Omega_{t+1}\right)\right) .
\end{aligned}
$$

Lemma 3.26 together with Bertsekas and Shreve (2004), Lemma 7.30(4), page 177 imply that $\hat{i}_{t}$ is u.s.a. Finally, as $\left\{\left(\omega^{t}, h, x, P\right), P \in \mathcal{Q}_{t+1}\left(\omega^{t}\right)\right\}$ is analytic [see Assumption 1.1], Bertsekas and Shreve (2004), Proposition 7.47, page 179, Lemma 7.30(4), page 178 apply and recalling (20) and (35), we get that $I_{t}\left(\omega^{t}, x\right)=$ $1_{[0, \infty)}(x) \sup _{h \in \mathbb{R}^{d}} \sup _{P \in \mathcal{Q}_{t+1}\left(\omega^{t}\right)} \hat{\mathbf{1}}_{t}\left(\omega^{t}, h, x, P\right)$ is u.s.a. and (27) for $t$ is proved.

For later purposes, we set $\overline{1}_{t}: \Omega^{t} \times \mathbb{R}^{d} \times[0, \infty) \times \mathfrak{P}\left(\Omega_{t+1}\right) \rightarrow \mathbb{R} \cup\{ \pm \infty\}$

$$
\overline{\mathbf{1}}_{t}\left(\omega^{t}, h, x, P\right):=\hat{\mathbf{1}}_{t}\left(\omega^{t}, h, x, P\right)+(-\infty) 1_{\mathbb{R}^{d} \backslash H_{x}^{t+1}\left(\omega^{t}, P\right)}(h) .
$$

Using Lemma 3.26, $\overline{1}_{t}$ is u.s.a. and

$$
\bar{I}_{t}\left(\omega^{t}, x\right):=1_{[0, \infty)}(x) \sup _{h \in \mathbb{R}^{d}} \sup _{P \in \mathcal{Q}_{t+1}\left(\omega^{t}\right)} \overline{1}_{t}\left(\omega^{t}, h, x, P\right)
$$

is u.s.a. as before. Furthermore, as $\hat{1}_{t} \geq 0$ we have that $\bar{I}_{t}=I_{t}$. To prove (25) and (28) at $t$, we apply Lemma 3.19 to $V\left(\omega_{t+1}, x\right)=U_{t+1}\left(\omega^{t}, \omega_{t+1}, x\right), I\left(\omega_{t+1}, x\right)=$ $I_{t+1}\left(\omega^{t}, \omega_{t+1}, x+1\right)[$ recall $(20)]$ and $\mathcal{G}=\mathcal{B}_{c}\left(\Omega_{t+1}\right)$ for any fixed $\omega^{t} \in \Omega^{t}$. Indeed

\footnotetext{
${ }^{4}$ As already mentioned, Bertsekas and Shreve (2004), Proposition 7.48, page 180, relies on Bertsekas and Shreve (2004), Lemma 7.30(4), page 177, applied for upper-semianalytic functions where the convention $-\infty+\infty=-\infty$ needs to be used. But here, as we deal with a nonnegative function the convention is useless.
} 
we have already proved [see the proof of (24) at $t$ ] that Assumption 3.12 holds true for $V$. From $(25)$ and $(28)$ at $t+1, I\left(\omega_{t+1}, \cdot\right)$ is nondecreasing and nonnegative on $\mathbb{R}$ for all $\omega_{t+1}$ and $V \leq I$. Finally, using Assumption 1.2 and (27) at $t+1$ together with Bertsekas and Shreve (2004), Lemma 7.30, page 177, we get that $\omega_{t+1} \rightarrow I_{t+1}\left(\omega^{t}, \omega_{t+1}, x+1+h \Delta S_{t+1}\left(\omega^{t}, \omega_{t+1}\right)\right)$ is $\mathcal{B}_{c}\left(\Omega_{t+1}\right)$-measurable.

We prove now (30) at $t$. Fix some $r \in \mathbb{Q}, r>0$. We have from the definition of $U_{t}$ [see (18), and (19)], (30) at $t+1$ and the definition of $J_{t}^{r}$ [see (22)] that for all $\omega^{t} \in \Omega^{t}$

$$
\begin{aligned}
U_{t}\left(\omega^{t}, r\right) & \geq \mathcal{U}_{t}\left(\omega^{t}, r\right) \geq \inf _{P \in \mathcal{Q}_{t+1}\left(\omega^{t}\right)} \int_{\Omega_{t+1}} U_{t+1}\left(\omega^{t}, \cdot, r\right) d P \\
& \geq \inf _{P \in \mathcal{Q}_{t+1}\left(\omega^{t}\right)} \int_{\Omega_{t+1}}-J_{t+1}^{r}\left(\omega^{t}, \cdot\right) d P=-J_{t}^{r}\left(\omega^{t}\right) .
\end{aligned}
$$

We prove now (29) at $t$. Choose $x \geq 0,\left(\phi_{s}\right)_{1 \leq s \leq t-1}$ universally predictable random variables and set $\bar{G}:=x+\sum_{s=1}^{t-1} \phi_{s} \Delta S_{s}$. Furthermore, fix some $P \in \mathcal{Q}^{t}$, $\xi \in \phi_{t}(\bar{G}, P), \varepsilon>0$ and set $G(\cdot):=\bar{G}(\cdot)+\xi(\cdot) \Delta S_{t}(\cdot)$. We apply Bertsekas and Shreve (2004), Proposition 7.50, page 184 , to $\overline{1}_{t}$ [see (36)] in order to obtain $S^{\varepsilon}:\left(\omega^{t}, x\right) \rightarrow\left(h^{\varepsilon}\left(\omega^{t}, x\right), p^{\varepsilon}\left(\cdot, \omega^{t}, x\right)\right) \in \mathbb{R}^{d} \times \mathfrak{P}\left(\Omega_{t+1}\right)$ that is analytically measurable such that $p^{\varepsilon}\left(\cdot, \omega^{t}, x\right) \in \mathcal{Q}_{t+1}\left(\omega^{t}\right)$ for all $\omega^{t} \in \Omega^{t}, x \geq 0$ and (recall that $\left.\bar{I}_{t}=I_{t}\right)$

$$
\overline{\mathbf{1}}_{t}\left(\omega^{t}, h^{\varepsilon}\left(\omega^{t}, x\right), x, p^{\varepsilon}\left(\cdot, \omega^{t}, x\right)\right) \geq \begin{cases}\frac{1}{\varepsilon} & \text { if } I_{t}\left(\omega^{t}, x\right)=\infty, \\ I_{t}\left(\omega^{t}, x\right)-\varepsilon & \text { otherwise. }\end{cases}
$$

Set $h_{G}^{\varepsilon}\left(\omega^{t}\right):=h^{\varepsilon}\left(\omega^{t}, 1_{\{G \geq 0\}}\left(\omega^{t}\right) G\left(\omega^{t}\right)\right), p_{G}^{\varepsilon}\left(\cdot, \omega^{t}\right):=p^{\varepsilon}\left(\cdot, \omega^{t}, 1_{\{G \geq 0\}}\left(\omega^{t}\right) G\left(\omega^{t}\right)\right)$. Using Bertsekas and Shreve (2004), Proposition 7.44, page 172, both $h_{G}^{\varepsilon}$ and $p_{G}^{\varepsilon}$ are $\mathcal{B}_{c}\left(\Omega^{t}\right)$-measurable. For some $\omega^{t} \in \Omega^{t}, y \geq 0$ fixed, if $h^{\varepsilon}\left(\omega^{t}, y\right) \notin$ $H_{y}^{t+1}\left(\omega^{t}, p^{\varepsilon}\left(\cdot, \omega^{t}, y\right)\right)$, using (36), we have $\overline{\mathbf{1}}_{t}\left(\omega^{t}, h^{\varepsilon}\left(\omega^{t}, y\right), y, p^{\varepsilon}\left(\cdot, \omega^{t}, y\right)\right)=$ $-\infty<\min \left(\frac{1}{\varepsilon}, I_{t}\left(\omega^{t}, y\right)-\varepsilon\right)$ [indeed from (25) at $t, I_{t} \geq 0$ ]. This contradicts (37) and, therefore, $h^{\varepsilon}\left(\omega^{t}, y\right) \in H_{y}^{t+1}\left(\omega^{t}, p^{\varepsilon}\left(\cdot, \omega^{t}, y\right)\right)$ and also $h_{G}^{\varepsilon}\left(\omega^{t}\right) \in$ $H_{G\left(\omega^{t}\right)}^{t+1}\left(\omega^{t}, p_{G}^{\varepsilon}\left(\cdot, \omega^{t}\right)\right)$ for $\omega^{t} \in\{G \geq 0\}$. We set $P_{G}^{\varepsilon}:=P \otimes p_{G}^{\varepsilon} \in \mathcal{Q}^{t+1}$ [see (2)] and get that

$$
\begin{aligned}
& P_{G}^{\varepsilon}\left(G(\cdot)+h_{G}^{\varepsilon}(\cdot) \Delta S_{t+1}(\cdot) \geq 0\right) \\
& \quad=\int_{\{G \geq 0\}} \int_{\Omega_{t+1}} p_{G}^{\varepsilon}\left(G\left(\omega^{t}\right)+h_{G}^{\varepsilon}\left(\omega^{t}\right) \Delta S_{t+1}\left(\omega^{t}, \omega_{t+1}\right) \geq 0, \omega^{t}\right) P\left(d \omega^{t}\right)=1,
\end{aligned}
$$

since $\{G \geq 0\}$ is a $\mathcal{Q}^{t}$-full measure set, $h_{G}^{\varepsilon} \in \phi_{t+1}\left(G, P_{G}^{\varepsilon}\right)$ follows. Using (35) and (36),

$$
\begin{aligned}
& \int_{\Omega^{t}} \overline{\mathrm{1}}_{t}\left(\omega^{t}, h_{G}^{\varepsilon}\left(\omega^{t}\right), p_{G}^{\varepsilon}\left(\omega^{t}\right), G\left(\omega^{t}\right)\right) P\left(d \omega^{t}\right) \\
& \quad=\int_{\Omega^{t+1}} I_{t+1}\left(\omega^{t+1}, G\left(\omega^{t}\right)+1+h_{G}^{\varepsilon}\left(\omega^{t}\right) \Delta S_{t+1}\left(\omega^{t+1}\right)\right) P_{G}^{\varepsilon}\left(d \omega^{t+1}\right) \leq A,
\end{aligned}
$$


where

$$
A:=\sup _{P \in \mathcal{Q}^{t+1}} \sup _{\xi \in \phi_{t+1}(G+1, P)} \int_{\Omega^{t+1}} I_{t+1}\left(\omega^{t+1}, G\left(\omega^{t}\right)+1+\xi\left(\omega^{t}\right) \Delta S_{t+1}\left(\omega^{t+1}\right)\right) P\left(d \omega^{t+1}\right)
$$

and $A<\infty$ using (29) at $t+1$ [note that $\left.\phi_{t+1}(G, P) \subset \phi_{t+1}(G+1, P)\right]$. Combining with (37), we find that

$$
\begin{gathered}
\frac{1}{\varepsilon} \int_{\left\{I_{t}(\cdot, G(\cdot))=\infty\right\}} P\left(d \omega^{t}\right)+\int_{\left\{I_{t}(\cdot, G(\cdot))<\infty\right\}}\left(I_{t}\left(\omega^{t}, G\left(\omega^{t}\right)\right)-\varepsilon\right) P\left(d \omega^{t}\right) \\
\leq \int_{\Omega^{t}} \overline{1}_{t}\left(\omega^{t}, h_{G}^{\varepsilon}\left(\omega^{t}\right), G\left(\omega^{t}\right), p_{G}^{\varepsilon}\left(\cdot, \omega^{t}\right)\right) P\left(d \omega^{t}\right) \leq A<\infty .
\end{gathered}
$$

As this is true for all $\varepsilon>0, P\left(\left\{I_{t}(\cdot, G(\cdot))=\infty\right\}\right)=0$ follows. Using again (38), we get that $\int_{\Omega^{t}} I_{t}\left(\omega^{t}, \bar{G}\left(\omega^{t-1}\right)+\xi\left(\omega^{t-1}\right) \Delta S_{t}\left(\omega^{t}\right)\right) P\left(d \omega^{t}\right) \leq A$ and as this is true for all $P \in \mathcal{Q}^{t}$ and $\xi \in \phi_{t}(\bar{G}, P),(29)$ is true for $t$.

We are left with the proof of (33) for $U_{t}$. Let $X=x+\sum_{s=1}^{t-1} \phi_{s} \Delta S_{s+1}$, with $x \geq 0$ and $\left(\phi_{s}\right)_{1 \leq s \leq t-1}$ some universally predictable random variables, be fixed such that $X \geq 0 \mathcal{Q}^{t}$-q.s. Let $\Omega_{X}^{t}:=\widetilde{\Omega}^{t} \cap\{X(\cdot) \geq 0\}$. Then $\Omega_{X}^{t} \in \mathcal{B}_{c}\left(\Omega^{t}\right)$ is a $\mathcal{Q}^{t}$ full measure set. We introduce the following random set $\psi_{X}: \Omega^{t} \rightarrow \mathbb{R}^{d}$ :

$$
\begin{aligned}
\psi_{X}\left(\omega^{t}\right):= & \left\{h \in \mathcal{D}_{X\left(\omega^{t}\right)}^{t+1}\left(\omega^{t}\right), U_{t}\left(\omega^{t}, X\left(\omega^{t}\right)\right)\right. \\
= & \left.\inf _{P \in \mathcal{Q}_{t+1}\left(\omega^{t}\right)} \int_{\Omega^{t+1}} U_{t+1}\left(\omega^{t}, \cdot, X\left(\omega^{t}\right)+h \Delta S_{t+1}\left(\omega^{t}, \cdot\right)\right) d P\right\}
\end{aligned}
$$

for $\omega^{t} \in \Omega_{X}^{t}$ and $\psi_{X}\left(\omega^{t}\right)=\varnothing$, otherwise $\left[\mathcal{D}_{X\left(\omega^{t}\right)}^{t+1}\left(\omega^{t}\right)\right.$ is defined in (17)]. To prove (33), it is enough to find some $\mathcal{B}_{c}\left(\Omega^{t}\right)$-measurable selector for $\psi_{X}$ and to show that $\Omega_{X}^{t} \subset\left\{\psi_{X} \neq \varnothing\right\}$. The last point follows from Proposition 3.28 and Theorem 3.23 [see (13), (14), (18), (19) and recall that $\Omega_{X}^{t} \subset \widetilde{\Omega}^{t}$ ]. Let $u_{X}: \Omega^{t} \times \mathbb{R}^{d} \rightarrow$ $\mathbb{R} \cup\{ \pm \infty\}$ be defined by [recall (34)] $u_{X}\left(\omega^{t}, h\right)=1_{\Omega_{X}^{t}}\left(\omega^{t}\right) \tilde{u}_{t}\left(\omega^{t}, h, X\left(\omega^{t}\right)\right)$. Using Rockafellar and Wets (1998), Proposition 14.39, page 666, Corollary 14.34, page 664 , we first prove that $-u_{X}$ is a $\mathcal{B}_{c}\left(\Omega^{t}\right)$-normal integrand [see Rockafellar and Wets (1998), Definition 14.27, page 661] and that $u_{X}$ is $\mathcal{B}_{c}\left(\Omega^{t}\right) \otimes \mathcal{B}\left(\mathbb{R}^{d}\right)$ measurable. Indeed we show that for all $h \in \mathbb{R}^{d}, u_{X}(\cdot, h)$ is $\mathcal{B}_{c}\left(\Omega^{t}\right)$-measurable and for all $\omega^{t} \in \Omega^{t}, u_{X}\left(\omega^{t}, \cdot\right)$ is u.s.c. and concave. The first point follows from the fact that $\tilde{u}_{t}$ is 1.s.a., $X$ is $\mathcal{B}_{c}\left(\Omega^{t}\right)$-measurable, $\Omega_{X}^{t} \in \mathcal{B}_{c}\left(\Omega^{t}\right)$ and Bertsekas and Shreve (2004), Proposition 7.44, page 172. Now we fix $\omega^{t} \in \Omega^{t}$. If $\omega^{t} \notin \Omega_{X}^{t}$, it is clear that $u_{X}\left(\omega^{t}, \cdot\right)$ is u.s.c. and concave. If $\omega^{t} \in \Omega_{X}^{t} \subset \widetilde{\Omega}^{t}$, we know from Proposition 3.28 that Lemma 3.22 applies and that $\phi_{\omega^{t}}(\cdot, \cdot)$ is u.s.c. and concave where

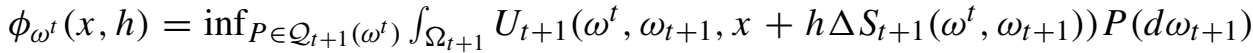
if $x \geq 0$ and $h \in \mathcal{H}_{x}^{t+1}\left(\omega^{t}\right)$ and $-\infty$ otherwise. In particular for $\omega^{t} \in \Omega_{X}^{t}$ and $x=X\left(\omega^{t}\right)$, we get that $\phi_{\omega^{t}}\left(X\left(\omega^{t}\right), \cdot\right)=u_{X}\left(\omega^{t}, \cdot\right)$ is u.s.c. and concave. Now, from the definitions of $\psi_{X}$ and $u_{X}$ for $\omega^{t} \in \Omega_{X}^{t}$, we have that

$$
\psi_{X}\left(\omega^{t}\right)=\left\{h \in \mathcal{D}_{X\left(\omega^{t}\right)}^{t+1}\left(\omega^{t}\right), U_{t}\left(\omega^{t}, X\left(\omega^{t}\right)\right)=u_{X}\left(\omega^{t}, h\right)\right\} .
$$


Lemma 3.26 implies that $\operatorname{Graph}\left(\mathcal{D}_{X}^{t+1}\right) \in \mathcal{B}_{c}\left(\Omega^{t}\right) \otimes \mathcal{B}\left(\mathbb{R}^{d}\right)$. Since $U_{t}$ is 1.s.a., $U_{t}$ is $\mathcal{B}_{c}\left(\Omega^{t} \times \mathbb{R}\right)$-measurable and Bertsekas and Shreve (2004), Lemma 7.29, page 174, implies that $U_{t}(\cdot, x)$ is $\mathcal{B}_{c}\left(\Omega^{t}\right)$-measurable for $x \in \mathbb{R}$ fixed. From $(24), U_{t}\left(\omega^{t}, \cdot\right)$ is u.s.c. and nondecreasing for any fixed $\omega^{t} \in \Omega^{t}$, so Blanchard, Carassus and Rásonyi (2016), Lemmata 7.12, 7.16, imply that $U_{t}$ is $\mathcal{B}_{c}\left(\Omega^{t}\right) \otimes \mathcal{B}(\mathbb{R})$-measurable. As $X$ is $\mathcal{B}_{c}\left(\Omega^{t}\right)$-measurable, we obtain that $U_{t}(\cdot, X(\cdot))$ is $\mathcal{B}_{c}\left(\Omega^{t}\right)$-measurable [see Bertsekas and Shreve (2004), Proposition 7.44, page 172]. It follows that $\operatorname{Graph}\left(\psi_{X}\right) \in \mathcal{B}_{c}\left(\Omega^{t}\right) \otimes \mathcal{B}\left(\mathbb{R}^{d}\right)$, we can apply the projection theorem [see Castaing and Valadier (1977), Theorem 3.23, page 75] and we get that $\left\{\psi_{X} \neq \varnothing\right\} \in \mathcal{B}_{c}\left(\Omega^{t}\right)$. Using the Auman theorem [see Sainte-Beuve (1974), Corollary 1], there exists some $\mathcal{B}_{c}\left(\Omega^{t}\right)$-measurable $\widehat{h}_{t+1}^{X}:\left\{\psi_{X} \neq \varnothing\right\} \rightarrow \mathbb{R}^{d}$ such that for all $\omega^{t} \in\left\{\psi_{X} \neq \varnothing\right\}$, $\widehat{h}_{t+1}^{X}\left(\omega^{t}\right) \in \psi_{X}\left(\omega^{t}\right)$. This concludes the proof of (33) extending $\widehat{h}_{t+1}^{X}$ on all $\Omega^{t}$ $\left(\widehat{h}_{t+1}^{X}=0\right.$ on $\left.\Omega^{t} \backslash\left\{\psi_{X} \neq \varnothing\right\}\right)$.

Proof of TheOREM 1.11. We proceed in three steps. First, we handle some integrability issues that are essential to the proof and were not required in Nutz (2016). In particular, we show that it is possible to apply the Fubini theorem. Then we build by induction a candidate for the optimal strategy, and finally we establish its optimality. The proof of the two last steps is very similar to the one of Nutz (2016).

Integrability issues. First, from Proposition 3.25 and (4), $u(x) \leq M_{x}<\infty$. We fix some $x \geq 0$ and $\phi \in \Phi\left(x, \mathcal{Q}^{T}\right)=\Phi\left(x, U, \mathcal{Q}^{T}\right)$; see again Proposition 3.25. From Proposition 3.29, we can apply by backward induction Proposition 3.30 for $t=T-1, T-2, \ldots, 0$. In particular, we get that (28) and (29) hold true for all $0 \leq t \leq T$ and choosing $G=V_{t-1}^{x+1, \phi}$ and $\xi=\phi_{t}$ [use Lemma A.33 since $\phi \in$ $\left.\Phi\left(x, \mathcal{Q}^{T}\right)\right]$, we get for all $P \in \mathcal{Q}^{t}$,

$$
\int_{\Omega^{t}} U_{t}^{+}\left(\omega^{t}, V_{t}^{x, \phi}\left(\omega^{t}\right)\right) P\left(d \omega^{t}\right)<\infty .
$$

So for all $P=P_{t-1} \otimes p \in \mathcal{Q}^{t}$ [see (2)] Bertsekas and Shreve (2004), Proposition 7.45 , page 175 , implies that

(40) $\int_{\Omega^{t}} U_{t}\left(\omega^{t}, V_{t}^{x, \phi}\left(\omega^{t}\right)\right) P\left(d \omega^{t}\right)=\int_{\Omega^{t-1}} \int_{\Omega_{t}} U_{t}\left(\omega^{t-1}, \omega_{t}, V_{t}^{x, \phi}\left(\omega^{t-1}, \omega_{t}\right)\right) p\left(d \omega_{t}, \omega^{t-1}\right) P_{t-1}\left(d \omega^{t-1}\right)$.

Construction of $\phi^{*}$. We fix some $x \geq 0$ and build by induction our candidate $\phi^{*}$ for the optimal strategy which will verify that

(41) $U_{t}\left(\omega^{t}, V_{t}^{x, \phi^{*}}\left(\omega^{t}\right)\right)=\inf _{P \in \mathcal{Q}_{t+1}\left(\omega^{t}\right)} \int_{\Omega_{t+1}} U_{t+1}\left(\omega^{t}, \cdot, V_{t}^{x, \phi^{*}}\left(\omega^{t}\right)+\phi_{t+1}^{*}\left(\omega^{t}\right) \Delta S_{t+1}\left(\omega^{t}, \cdot\right)\right) d P$.

We start at $t=0$ and use (33) in Proposition 3.30 with $X=x \geq 0$. We set $\phi_{1}^{*}:=\widehat{h}_{1}^{x} \in \mathcal{D}_{x}^{1}$ and we obtain that $P_{1}\left(x+\phi_{1}^{*} \Delta S_{1}(\cdot) \geq 0\right)=1$ for all $P \in \mathcal{Q}^{1}$ and that (41) holds true for $t=0$. Assume that until some $t \geq 1$ we have found some universally predictable random variables $\left(\phi_{s}^{*}\right)_{1 \leq s \leq t}$ and some sets $\left(\bar{\Omega}^{s}\right)_{1 \leq s \leq t-1}$ 
such that $\bar{\Omega}^{s} \in \mathcal{B}_{c}\left(\Omega^{s}\right)$ is a $\mathcal{Q}^{s}$-full measure set, $\phi_{s+1}^{*}\left(\omega^{s}\right) \in D^{s+1}\left(\omega^{s}\right)$ for all $\omega^{s} \in \bar{\Omega}^{s},\left\{V_{s+1}^{x, \phi^{*}}(\cdot) \geq 0\right\}$ is a $Q^{s+1}$-full measure set and (41) holds true at $s$ for all $\omega^{s} \in \bar{\Omega}^{s}$ where $s=0, \ldots, t-1$. We apply Proposition 3.30 with $X=V_{t}^{x, \phi^{*}}$ and there exists $\mathcal{Q}^{t}$-full measure set $\bar{\Omega}^{t}:=\Omega_{V_{t}^{x, \phi^{*}}}^{t} \in \mathcal{B}_{c}\left(\Omega^{t}\right)$ and some $\mathcal{B}_{c}\left(\Omega^{t}\right)$ measurable random variable $\phi_{t+1}^{*}:=\widehat{h}_{t+1}^{V_{t}^{x, \phi^{*}}}$ such that $\phi_{t+1}^{*}\left(\omega^{t}\right) \in \mathcal{D}_{V_{t}^{x, \phi^{*}}\left(\omega^{t}\right)}^{t+1}\left(\omega^{t}\right)$ for all $\omega^{t} \in \bar{\Omega}^{t}$ and (41) holds true at $t$. Let $P^{t+1}=P \otimes p \in \mathcal{Q}^{t+1}$ where $P \in \mathcal{Q}^{t}$ and $p \in \mathcal{S} K_{t+1}$ with $p\left(\cdot, \omega^{t}\right) \in \mathcal{Q}_{t+1}\left(\omega^{t}\right)$ for all $\omega^{t} \in \bar{\Omega}^{t}$ [see (2)]. From Bertsekas and Shreve (2004), Proposition 7.45, page 175, we get

$$
P_{t+1}\left(V_{t+1}^{x, \phi^{*}} \geq 0\right)=\int_{\Omega^{t}} p\left(V_{t}^{x, \phi^{*}}\left(\omega^{t}\right)+\phi_{t+1}^{*}\left(\omega^{t}\right) \Delta S_{t+1}\left(\omega^{t}, \cdot\right) \geq 0, \omega^{t}\right) P\left(d \omega^{t}\right)=1,
$$

where we have used that $\phi_{t+1}^{*}\left(\omega^{t}\right) \in \mathcal{H}_{V_{t}^{x, \phi^{*}}\left(\omega^{t}\right)}^{t+1}\left(\omega^{t}\right)$ for all $\omega^{t} \in \bar{\Omega}^{t}$ and $P\left(\bar{\Omega}^{t}\right)=1$ and we can continue the recursion. Thus, we have found that $\phi^{*} \in \Phi\left(x, \mathcal{Q}^{T}\right)$ and from Proposition 3.25, $\phi^{*} \in \Phi\left(x, U, \mathcal{Q}^{T}\right)$.

Optimality of $\phi^{*}$. We fix some $P=P_{T-1} \otimes p_{T} \in \mathcal{Q}^{T}$. Using (40), $P_{T-1}\left(\bar{\Omega}^{T-1}\right)=1$ and (41) for $t=T-1$ we get that

$$
\begin{aligned}
& E_{P} U\left(\cdot, V_{T}^{x, \phi^{*}}(\cdot)\right) \\
& \quad=\int_{\bar{\Omega}^{T-1}} \int_{\Omega_{T}} U_{T}\left(\omega^{T-1}, \omega_{T}, V_{T-1}^{x, \phi^{*}}\left(\omega^{T-1}\right)+\phi_{T}^{*}\left(\omega^{T-1}\right) \Delta S_{T}\left(\omega^{T-1}, \omega_{T}\right)\right) p_{T}\left(d \omega_{T}, \omega^{T-1}\right) P_{T-1}\left(d \omega^{T-1}\right) \\
& \geq \int_{\Omega^{T-1}} U_{T-1}\left(\omega^{T-1}, V_{T-1}^{x, \phi^{*}}\left(\omega^{T-1}\right)\right) P_{T-1}\left(d \omega^{T-1}\right) .
\end{aligned}
$$

We iterate the process by backward induction and obtain that (recall that $\Omega^{0}:=$ $\left.\left\{\omega_{0}\right\}\right) U_{0}(x) \leq E_{P} U\left(\cdot, V_{T}^{x, \phi^{*}}(\cdot)\right)$. As the preceding equality holds true for all $P \in$ $\mathcal{Q}^{T}$ and as $\phi^{*} \in \Phi\left(x, U, \mathcal{Q}^{T}\right)$, we get that $U_{0}(x) \leq u(x)$ [see (4)]. So $\phi^{*}$ will be optimal if $U_{0}(x) \geq u(x)$. We fix some $\phi \in \Phi\left(x, U, \mathcal{Q}^{T}\right)$ and show that

$$
\inf _{P \in \mathcal{Q}^{t+1}} E_{P} U_{t+1}\left(\cdot, V_{t+1}^{x, \phi}(\cdot)\right) \leq \inf _{Q \in \mathcal{Q}^{t}} E_{Q} U_{t}\left(\cdot, V_{t}^{x, \phi}(\cdot)\right), \quad t \in\{0, \ldots, T-1\} .
$$

Then $\inf _{P \in \mathcal{Q}^{T}} E_{P} U_{T}\left(\cdot, V_{T}^{x, \phi}(\cdot)\right) \leq \inf _{Q \in \mathcal{Q}^{1}} E_{Q} U_{1}\left(\cdot, V_{1}^{x, \phi}(\cdot)\right) \leq U_{0}(x)$ is obtained recursively [recall (31)]. As this is true for all $\phi \in \Phi\left(x, U, \mathcal{Q}^{T}\right), u(x) \leq U_{0}(x)$ and the proof is complete.

We fix some $t \in\{0, \ldots, T-1\}$ and prove (42). As $U_{t+1}$ is 1.s.a. [see (26)] and Assumption 1.2 holds true, Bertsekas and Shreve (2004), Lemma 7.30(3), page 177, Proposition 7.48, page 180, imply that $f$ is l.s.a. where

$$
f\left(\omega^{t}, y, h, P\right):=\int_{\Omega_{t+1}} U_{t+1}\left(\omega^{t}, \cdot, y+h \Delta S_{t+1}\left(\omega^{t}, \cdot\right)\right) d P .
$$

Let $f^{*}\left(\omega^{t}, y, h\right)=\inf _{P \in \mathcal{Q}_{t+1}\left(\omega^{t}\right)} f\left(\omega^{t}, y, h, P\right)$ and fix some $\varepsilon>0$. Then since $\left\{\left(\omega^{t}, y, h, P\right), P \in \mathcal{Q}_{t+1}\left(\omega^{t}\right)\right\}$ is an analytic set [recall Assumption 1.1], Bertsekas 
and Shreve (2004), Proposition 7.50, page 184, implies that there exists some universally measurable $\widetilde{p}_{t+1}^{\varepsilon}:\left(\omega^{t}, y, h\right) \rightarrow \mathfrak{P}\left(\Omega_{t+1}\right)$ such that $\tilde{p}_{t+1}^{\varepsilon}\left(\cdot, \omega^{t}, y, h\right) \in$ $\mathcal{Q}_{t+1}\left(\omega^{t}\right)$ for all $\left(\omega^{t}, y, h\right) \in \Omega^{t} \times \mathbb{R} \times \mathbb{R}^{d}$ and

(43) $f\left(\omega^{t}, y, h, \widetilde{p}_{t+1}^{\varepsilon}\left(\cdot, \omega^{t}, y, h\right)\right) \leq \begin{cases}f^{*}\left(\omega^{t}, y, h\right)+\varepsilon & \text { if } f^{*}\left(\omega^{t}, y, h\right)>-\infty, \\ -\frac{1}{\varepsilon} & \text { otherwise. }\end{cases}$

Let $p_{t+1}^{\varepsilon}\left(\cdot, \omega^{t}\right)=\tilde{p}_{t+1}^{\varepsilon}\left(\cdot, \omega^{t}, V_{t}^{x, \phi}\left(\omega^{t}\right), \phi_{t+1}\left(\omega^{t}\right)\right)$ : Bertsekas and Shreve (2004), Proposition 7.44, page 172, implies that $p_{t+1}^{\varepsilon}$ is $\mathcal{B}_{c}\left(\Omega^{t}\right)$-measurable. For all $\omega^{t} \in$ $\widetilde{\Omega}^{t} \cap\left\{V_{t}^{x, \phi}(\cdot) \geq 0\right\}$,

$$
\begin{aligned}
f^{*}\left(\omega^{t}, V_{t}^{x, \phi}\left(\omega^{t}\right), \phi_{t+1}\left(\omega^{t}\right)\right) & \leq \sup _{h \in \mathcal{H}_{V_{t}^{x+\phi}\left(\omega^{t}\right)}^{t+1}} f^{*}\left(\omega^{t}, V_{t}^{x, \phi}\left(\omega^{t}\right), h\right) \\
& =U_{t}\left(\omega^{t}, V_{t}^{x, \phi}\left(\omega^{t}\right)\right)
\end{aligned}
$$

[use Lemma A.33 since $\phi \in \Phi\left(x, \mathcal{Q}^{T}\right)$ and recall (31)]. Choosing $y=V_{t}^{x, \phi}\left(\omega^{t}\right)$, $h=\phi_{t+1}\left(\omega^{t}\right)$ in (43), we find that for all $\omega^{t} \in \widetilde{\Omega}^{t} \cap\left\{V_{t}^{x, \phi}(\cdot) \geq 0\right\}$

$$
\begin{gathered}
\int_{\Omega_{t+1}} U_{t+1}\left(\omega^{t}, \omega_{t+1}, V_{t+1}^{x, \phi}\left(\omega^{t}, \omega_{t+1}\right)\right) p_{t+1}^{\varepsilon}\left(d \omega_{t+1}, \omega^{t}\right)-\varepsilon \\
\leq \max \left(U_{t}\left(\omega^{t}, V_{t}^{x, \phi}\left(\omega^{t}\right)\right),-\frac{1}{\varepsilon}-\varepsilon\right) .
\end{gathered}
$$

Fix some $Q \in \mathcal{Q}^{t}$ and set $P^{\varepsilon}:=Q \otimes p_{t+1}^{\varepsilon} \in \mathcal{Q}^{t+1}$ [see (2)]. Using (40) and since $\widetilde{\Omega}^{t} \cap\left\{V_{t}^{x, \phi}(\cdot) \geq 0\right\}$ is a $\mathcal{Q}^{t}$ full measure set [recall again that $\phi \in \Phi\left(x, \mathcal{Q}^{T}\right)$ and Lemma A.33], we get

$$
\begin{aligned}
\inf _{P \in \mathcal{Q}^{t+1}} E_{P} U_{t+1}\left(\cdot, V_{t+1}^{x, \phi}(\cdot)\right)-\varepsilon & \leq E_{P^{\varepsilon}} U_{t+1}\left(\cdot, V_{t+1}^{x, \phi}(\cdot)\right)-\varepsilon \\
& \leq E_{Q} \max \left(U_{t}\left(\cdot, V_{t}^{x, \phi}(\cdot)\right),-\frac{1}{\varepsilon}-\varepsilon\right) .
\end{aligned}
$$

Since for all $0<\varepsilon<1, \max \left(U_{t}\left(\cdot, V_{t}^{x, \phi}(\cdot)\right),-\frac{1}{\varepsilon}-\varepsilon\right) \leq-1+U_{t}^{+}\left(\cdot, V_{t}^{x, \phi}(\cdot)\right)$, recalling (39), letting $\varepsilon$ go to zero and applying Fatou's lemma, we obtain that $\inf _{P \in \mathcal{Q}^{t+1}} E_{P} U_{t+1}\left(\cdot, V_{t+1}^{x, \phi}(\cdot)\right) \leq E_{Q} U_{t}\left(\cdot, V_{t}^{x, \phi}(\cdot)\right)$. As this holds true for all $Q \in$ $\mathcal{Q}^{t},(42)$ is proved.

PROOF OF THEOREM 3.6. Since the $s N A\left(\mathcal{Q}^{T}\right)$ condition holds true, the $N A\left(\mathcal{Q}^{T}\right)$ condition is also verified and to apply Theorem 1.11 it remains to prove that Assumption 3.5 is satisfied. We fix some $P \in \mathcal{Q}^{T} x \geq 0$ and some $\phi \in \phi(x, P)$. Since the $N A(P)$ condition holds true, using similar arguments as in the proof of Blanchard, Carassus and Rásonyi (2016), Theorem 4.17, we find 
that for $P_{t}$-almost all $\omega^{t} \in \Omega^{t},\left|V_{t}^{x, \phi}\left(\omega^{t}\right)\right| \leq \prod_{s=1}^{t}\left(x+\frac{\left|\Delta S_{s}\left(\omega^{s}\right)\right|}{\alpha_{s-1}^{P}\left(\omega^{s-1}\right)}\right)$. Note that $V^{x, \phi}$ is universally adapted and that $\sup _{P \in \mathcal{Q}^{t}} E_{P}\left|V_{t}^{x, \phi}(\cdot)\right|^{r}<\infty$ for all $r>0$ [recall that $\Delta S_{s}, \frac{1}{\alpha_{s}^{P}} \in \mathcal{W}_{s}$ for all $s \geq 1$ ]. The monotonicity of $U^{+}$and Proposition 3.24 [with $\left.\lambda=2 \prod_{s=1}^{T}\left(1+\frac{\left|\Delta S_{s}\left(\omega^{s}\right)\right|}{\alpha_{s-1}^{P}\left(\omega^{s-1}\right)}\right) \geq 1\right]$ implies that for $P_{t}$-almost all $\omega^{t} \in \Omega^{t}$

$$
U^{+}\left(\omega^{T}, V_{T}^{1, \phi}\left(\omega^{T}\right)\right) \leq 4\left(\prod_{s=1}^{T}\left(1+\frac{\left|\Delta S_{S}\left(\omega^{s}\right)\right|}{\alpha_{s-1}^{P}\left(\omega^{s-1}\right)}\right)\right)\left(U^{+}\left(\omega^{T}, 1\right)+C_{T}\left(\omega^{T}\right)\right)
$$

We set $N:=4 \sup _{P \in \mathcal{Q}^{T}} E_{P}\left(\left(\prod_{s=1}^{T}\left(1+\frac{\left|\Delta S_{s}\left(\omega^{s}\right)\right|}{\alpha_{s-1}^{P}\left(\omega^{s-1}\right)}\right)\right)\left(U^{+}\left(\omega^{T}, 1\right)+C_{T}\left(\omega^{T}\right)\right)\right)$. Since $U^{+}(\cdot, 1), U^{-}\left(\cdot, \frac{1}{4}\right) \in \mathcal{W}_{T}$ and $\Delta S_{s}, \frac{1}{\alpha_{s}^{P}} \in \mathcal{W}_{s}$ for all $s \geq 1$, we obtain that $N<\infty$ (recall the definition of $C_{T}$ in Proposition 3.24). Using (44), we find that $E_{P} U^{+}\left(\cdot, V_{T}^{1, \phi}(\cdot)\right) \leq N<\infty$ and as this is true for all $P \in \mathcal{Q}^{T}$ and $\phi \in \Phi(1, P)$, Assumption 3.5 holds true.

\section{APPENDIX}

A.1. Auxiliary results. The two first lemmata were used in the proof of Theorem 3.23 and Lemma 3.26. The second one is a well-known result on concave functions in which the proof is given since we did not find some reference.

LEMMA A.31. Assume that Assumption 3.7 holds true. For all $x>0$, we have $\operatorname{Aff}\left(\mathcal{H}_{x}\right)=\mathbb{R}^{d}, \operatorname{Ri}\left(\mathcal{H}_{x}\right)$ is an open set in $\mathbb{R}^{d}$ and $\mathbb{Q}^{d}$ is dense in $\operatorname{Ri}\left(\mathcal{H}_{x}\right){ }^{5}$ Moreover, $\operatorname{Ri}\left(\mathcal{H}_{x}\right) \subset \bigcup_{r \in \mathbb{Q}, r>0} \mathcal{H}_{x}^{r} \subset \mathcal{H}_{x}$ and, therefore, $\bigcup_{r \in \mathbb{Q}, r>0} \mathcal{H}_{x}^{r}=\mathcal{H}_{x}$, where the closure is taken in $\mathbb{R}^{d}$. If furthermore, we assume that there exists some $0 \leq c<\infty$ such that $Y_{i}(\omega) \leq c$ for all $i=1, \ldots, d, \omega \in \bar{\Omega}$ (recalling Assumption $3.7,|Y|$ is bounded) then $\operatorname{Ri}\left(\mathcal{H}_{x}\right)=\bigcup_{r \in \mathbb{Q}, r>0} \mathcal{H}_{x}^{r}$.

Proof. Fix some $x>0$. Let $\varepsilon>0$ be such that $x-\varepsilon>0$ and $R:=\{h \in$ $\left.\mathbb{R}^{d}, 0 \leq h_{i} \leq \frac{x-\varepsilon}{d b}\right\}$. Using Assumption 3.7, if $h \in R$ for all $\omega \in \bar{\Omega}, x+h Y(\omega) \geq$ $x-b \sum_{i=1}^{d} h_{i} \geq \varepsilon$ and $h \in \mathcal{H}_{x}^{\varepsilon} \subset \mathcal{H}_{x}$. Thus $R \subset \mathcal{H}_{x}$ and $\operatorname{Aff}\left(\mathcal{H}_{x}\right)=\mathbb{R}^{d}$ follows (recall that $0 \in \mathcal{H}_{x}$ ). Therefore, $\operatorname{Ri}\left(\mathcal{H}_{x}\right)$ is the interior of $\mathcal{H}_{x}$ in $\mathbb{R}^{d}$, and thus an open set in $\mathbb{R}^{d}$ and the fact that $\mathbb{Q}^{d}$ is dense in $\operatorname{Ri}\left(\mathcal{H}_{x}\right)$ follows immediately. Fix now some $h \in \operatorname{Ri}\left(\mathcal{H}_{x}\right)$. As $0 \in \mathcal{H}_{x}$, there exists some $\varepsilon>0$ such that $(1+\varepsilon) h \in \mathcal{H}_{x}$ [see Rockafellar (1970), Theorem 6.4, page 47] which implies that $x+h Y(\cdot) \geq$ $\frac{\varepsilon}{1+\varepsilon} x>0 \mathcal{Q}$-q.s., hence $h \in \mathcal{H}_{x}^{r}$ for $r \in \mathbb{Q}$ such that $0<r \leq \frac{\varepsilon}{1+\varepsilon} x$ and $\operatorname{Ri}\left(\mathcal{H}_{x}\right) \subset$ $\bigcup_{r \in \mathbb{Q}, r>0} \mathcal{H}_{x}^{r} \subset \mathcal{H}_{x}$ is proved and also $\overline{\bigcup_{r \in \mathbb{Q}, r>0} \mathcal{H}_{x}^{r}}=\mathcal{H}_{x}$ since $\overline{\operatorname{Ri}\left(\mathcal{H}_{x}\right)}=\mathcal{H}_{x}$. Assume now that $|Y|$ is bounded by some constant $K>0$. Let $h \in \bigcup_{r \in \mathbb{Q}, r>0} \mathcal{H}_{x}^{r}$

\footnotetext{
${ }^{5}$ For a Polish space $X$, we say that a set $D \subset X$ is dense in $B \subset X$ if for all $\varepsilon>0, b \in B$, there exists $d \in D \cap B$ such that $d(b, d)<\varepsilon$ where $d$ is a metric on $X$ consistent with its topology.
} 
and $r \in \mathbb{Q}, r>0$ be such that $h \in \mathcal{H}_{x}^{r}$, we set $\varepsilon:=\frac{r}{2 K}$. Then for any $g \in B(0, \varepsilon)$, we have for $\mathcal{Q}$-almost all $\omega \in \bar{\Omega}$ that $x+(h+g) Y(\omega) \geq r+g Y(\omega) \geq r-|g||Y(\omega)| \geq$ $\frac{r}{2}$, hence $h+g \in \mathcal{H}_{x}, B(h, \varepsilon) \subset \mathcal{H}_{x}$ and $h$ belongs to the interior of $\mathcal{H}_{x}$ [and also to $\left.\operatorname{Ri}\left(\mathcal{H}_{x}\right)\right]$.

LEMMA A.32. Let $f: \mathbb{R}^{d} \rightarrow \mathbb{R} \cup\{ \pm \infty\}$ be a concave function such that $\operatorname{Ri}(\operatorname{Dom} f) \neq \varnothing$. Then $\sup _{h \in \operatorname{Dom} f} f(h)=\sup _{h \in \operatorname{Ri}(\operatorname{Dom} f)} f(h)$.

Proof. Let $C:=\sup _{h \in \operatorname{Ri}(\operatorname{Dom} f)} f(h)$ and $h_{1} \in \operatorname{Dom} f \backslash \operatorname{Ri}(\operatorname{Dom} f$ ) be fixed. We have to prove that $f\left(h_{1}\right) \leq C$. If $C=\infty$, there is nothing to show. So assume that $C<+\infty$. Let $h_{0} \in \operatorname{Ri}(\operatorname{Dom} f)$ and introduce $\phi: t \in \mathbb{R} \rightarrow f\left(t h_{1}+(1-t) h_{0}\right)$ if $t \in[0,1]$ and $-\infty$ otherwise. From Rockafellar (1970), Theorem 6.1, page 45, $t h_{1}+(1-t) h_{0} \in \operatorname{Ri}(\operatorname{Dom} f)$ if $t \in[0,1)$, and thus $[0,1) \subset\{t \in[0,1], \phi(t) \leq C\}$. Clearly, $\phi$ is concave on $\mathbb{R}$. Since Dom $f$ is convex, Dom $\phi=[0,1]$. So, using Föllmer and Schied (2002), Proposition A.4, page 400, $\phi$ is 1.s.c. on $[0,1]$ and $\{t \in[0,1], \phi(t) \leq C\}$ is a closed set in $\mathbb{R}$. It follows that $1 \in\{t \in[0,1], \phi(t) \leq C\}$, that is, $f\left(h_{1}\right) \leq C$ and the proof is complete.

The following lemma was used several times.

LEMmA A.33. Assume that the $N A\left(\mathcal{Q}^{T}\right)$ condition holds true. Let $\phi \in \Phi$ such that $V_{T}^{x, \phi} \geq 0 \mathcal{Q}^{T}$-q.s. [i.e., $\left.\phi \in \Phi\left(x, \mathcal{Q}^{T}\right)\right]$, then $V_{t}^{x, \phi} \geq 0 \mathcal{Q}^{t}$-q.s. for all $t \in$ $\{0, \ldots, T\}$.

ProOF. Let $\phi \in \Phi$ be such that $V_{T}^{x, \phi} \geq 0 \mathcal{Q}^{T}$-q.s. and assume that $V_{t}^{x, \phi} \geq 0$ $\mathcal{Q}^{t}$-q.s. for all $t$ does not hold true. Then $n:=\sup \left\{t, \exists P_{t} \in \mathcal{Q}^{t}, P_{t}\left(V_{t}^{x, \phi}<0\right)>\right.$ $0\}<T$ and there exists some $\widehat{P}_{n} \in \mathcal{Q}^{n}$ such that $\widehat{P}_{n}(A)>0$ where $A=\left\{V_{n}^{x, \phi}<\right.$ $0\} \in \mathcal{B}_{c}\left(\Omega^{n}\right)$ and for all $s \geq n+1, P \in \mathcal{Q}^{s}, P\left(V_{s}^{x, \phi} \geq 0\right)=1$. Let $\Psi_{s}\left(\omega^{s-1}\right)=0$ if $1 \leq s \leq n$ and $\Psi_{s}\left(\omega^{s-1}\right)=1_{A}\left(\omega^{n}\right) \phi_{s}\left(\omega^{s-1}\right)$ if $s \geq n+1$. Then $\Psi \in \Phi$ and $V_{T}^{0, \Psi}=$ $\sum_{k=n+1}^{T} \Psi_{s} \Delta S_{s}=1_{A}\left(V_{T}^{x, \phi}-V_{n}^{x, \phi}\right)$. Thus $V_{T}^{0, \Psi} \geq 0 \mathcal{Q}^{T}$-q.s. and $V_{T}^{0, \Psi}>0$ on $A$. Let $\widehat{P}_{T}:=\widehat{P}_{n} \otimes p_{n+1} \otimes \cdots \otimes p_{T} \in \mathcal{Q}^{T}$ where for $s=n+1, \cdot, T, p_{s}(\cdot, \cdot)$ is a given universally measurable selector of $\mathcal{Q}^{s}$ [see (2)]. It is clear that $\widehat{P}_{T}(A)=\widehat{P}_{n}(A)>0$, hence we get an arbitrage opportunity.

A.2. Measure theoretical issues. In this section, we first provide some counter-examples to Bouchard and Nutz (2015), Lemma 4.12, and propose an alternative to this lemma. Our counter-example A.34 is based on a result from Gelbaum and Olmsted (1964) originally due to Sierpinski (1920). An other counter-example can be found Rockafellar and Wets (1998), Proposition 14.28, page 661 . 
EXAMPLE A.34. We denote by $\mathcal{L}\left(\mathbb{R}^{2}\right)$ the Lebesgue sigma-algebra on $\mathbb{R}^{2}$. Recall that $\mathcal{B}\left(\mathbb{R}^{2}\right) \subset \mathcal{L}\left(\mathbb{R}^{2}\right)$. Let $A \notin \mathcal{L}\left(\mathbb{R}^{2}\right)$ be such that every line has at most two common points with $A$ [see Gelbaum and Olmsted (1964), Example 22, page 142, for the proof of the existence of $A]$ and define $F: \mathbb{R}^{2} \rightarrow \mathbb{R}$ by $F(x, y):=1_{A}(x, y)$. We fix some $x \in \mathbb{R}$ and let $A_{x}^{1}:=\{y \in \mathbb{R},(x, y) \in A\}$. By assumption, $A_{x}^{1}$ contains at most two points: thus it is a closed subset of $\mathbb{R}$. It follows that $\{y \in \mathbb{R}$, $F(x, y) \geq c\}$ is a closed subset of $\mathbb{R}$ for all $c \in \mathbb{R}$ and $F(x, \cdot)$ is u.s.c. Similarly, the function $F(\cdot, y)$ is u.s.c., and thus $\mathcal{B}(\mathbb{R})$-measurable for all $y \in \mathbb{R}$ fixed. But since $A \notin \mathcal{L}\left(\mathbb{R}^{2}\right), F$ is not $\mathcal{L}\left(\mathbb{R}^{2}\right)$-measurable and, therefore, not $\mathcal{B}(\mathbb{R}) \otimes \mathcal{B}(\mathbb{R})$ measurable.

We propose now the following correction to Bouchard and Nutz (2015), Lemma 4.12. Note that Lemma A.35 can be applied in the proof of Nutz (2016), Lemma 3.7, since the considered function is concave [as well as in the proof of Bouchard and Nutz (2015), Lemma 4.10, where the considered function is convex].

LEMMA A.35. Let $(A, \mathcal{A})$ be a measurable space and let $\theta: \mathbb{R}^{d} \times A \rightarrow \mathbb{R} \cup$ $\{ \pm \infty\}$ be a function such that $\omega \rightarrow \theta(y, \omega)$ is $\mathcal{A}$-measurable for all $y \in \mathbb{R}^{d}$ and $y \rightarrow \theta(y, \omega)$ is l.s.c. and convex for all $\omega \in A$. Then $\theta$ is $\mathcal{B}\left(\mathbb{R}^{d}\right) \otimes \mathcal{A}$-measurable.

PROOF. It is a direct application of Rockafellar and Wets (1998), Proposition 14.39, page 666, Corollary 14.34, page 664 .

We finish with three lemmata related to measurability issues used throughout the paper.

Lemma A.36. Let $X, Y$ be two Polish spaces and $F: X \times Y \rightarrow \mathbb{R} \cup\{ \pm \infty\}$ be u.s.a. [resp., l.s.a.]. Then, for $x \in X$ fixed, the function $F_{x}: y \in Y \rightarrow F(x, y) \in$ $\mathbb{R} \cup\{ \pm \infty\}$ is u.s.a. [resp., l.s.a.].

Proof. Assume that $F$ is u.s.a. and fix some $c \in \mathbb{R}$, then $C:=F^{-1}((c, \infty)) \in$ $\mathcal{A}(X \times Y)$. Fix now some $x \in X$. Since $I_{x}: y \rightarrow(x, y)$ is $\mathcal{B}(Y)$-measurable, applying Bertsekas and Shreve (2004), Proposition 7.40, page 165, we get that $\left\{y \in Y, F_{x}(y)>c\right\}=\{y \in Y,(x, y) \in C\}=I_{x}^{-1}(C) \in \mathcal{A}(Y)$.

LemMA A.37. Assume that Assumptions 1.1 and 1.2 hold true. Let $0 \leq t \leq$ $T-1, B \in \mathcal{B}(\mathbb{R})$. Then

$$
\begin{aligned}
F_{B}:\left(\omega^{t}, P, h, x\right) \rightarrow & P\left(x+h \Delta S_{t+1}\left(\omega^{t}, \cdot\right) \in B\right) \\
& \text { is } \mathcal{B}\left(\Omega^{t}\right) \otimes \mathcal{B}\left(\mathfrak{P}\left(\Omega_{t+1}\right)\right) \otimes \mathcal{B}\left(\mathbb{R}^{d}\right) \otimes \mathcal{B}(\mathbb{R}) \text {-measurable, } \\
H_{B}:\left(\omega^{t}, h, x\right) \rightarrow & \inf _{P \in \mathcal{Q}_{t+1}\left(\omega^{t}\right)} P\left(x+h \Delta S_{t+1}\left(\omega^{t}, \cdot\right) \in B\right) \in \mathcal{L} S A\left(\Omega^{t} \times \mathbb{R}^{d} \times \mathbb{R}\right),
\end{aligned}
$$




$$
K_{B}:\left(\omega^{t}, h\right) \rightarrow \sup _{P \in \mathcal{Q}_{t+1}\left(\omega^{t}\right)} P\left(x+h \Delta S_{t+1}\left(\omega^{t}, \cdot\right) \in B\right) \in \mathcal{U} S A\left(\Omega^{t} \times \mathbb{R}^{d}\right) .
$$

ProOF. The first assertion follows from Bertsekas and Shreve (2004), Proposition 7.29, page 144, applied to $f\left(\omega_{t+1}, \omega^{t}, P, h, x\right)=1_{x+h \Delta S_{t+1}\left(\omega^{t}, \cdot\right) \in B}\left(\omega_{t+1}\right)$ [recall Assumption 1.2] and $q\left(d \omega_{t+1} \mid \omega^{t}, P, h, x\right)=P\left(d \omega_{t+1}\right)$. The second one is obtained applying Bertsekas and Shreve (2004), Proposition 7.47, page 179, to $F_{B}$ [recall Assumption 1.1]. The last assertion is using $\sup _{P \in \mathcal{Q}_{t+1}\left(\omega^{t}\right)} P(x+$ $\left.h \Delta S_{t+1}\left(\omega^{t}, \cdot\right) \in B\right)=1-\inf _{P \in \mathcal{Q}_{t+1}\left(\omega^{t}\right)} P\left(x+h \Delta S_{t+1}\left(\omega^{t}, \cdot\right) \in B^{c}\right)$ and Lemma A.36.

LEMMA A.38. Let $X$ be a Polish space and $\Lambda$ be an $\mathbb{R}^{d}$-valued random variable:

(i) Assume that $\operatorname{Graph}(\Lambda) \in \mathcal{B}_{c}(X) \otimes \mathcal{B}\left(\mathbb{R}^{d}\right)$. Then $\operatorname{Graph}(\bar{\Lambda}) \in \mathcal{B}_{c}(X) \otimes$ $\mathcal{B}\left(\mathbb{R}^{d}\right)$ where $\bar{\Lambda}$ is defined by $\bar{\Lambda}(x)=\overline{\Lambda(x)}$ for all $x \in X$, where the closure is taken in $\mathbb{R}^{d}$.

(ii) Assume now that $\Lambda$ is open valued and $\operatorname{Graph}(\Lambda) \in \mathcal{C} A\left(X \times \mathbb{R}^{d}\right)$. Then $\operatorname{Graph}(\Lambda) \in \mathcal{B}_{c}(X) \otimes \mathcal{B}\left(\mathbb{R}^{d}\right)$.

PROOF. From Rockafellar and Wets (1998), Theorem 14.8, page 648, $\Lambda$ is $\mathcal{B}_{c}(X)$-measurable [see Rockafellar and Wets (1998), Definition 14.1, page 643] and using Aliprantis and Border (2006), Theorem 18.6, page 596, we get that $\operatorname{Graph}(\bar{\Lambda}) \in \mathcal{B}_{c}(X) \otimes \mathcal{B}\left(\mathbb{R}^{d}\right)$. Now we prove (ii). Fix some open set $O \subset \mathbb{R}^{d}$ and let $\Lambda^{c}(x)=\mathbb{R}^{d} \backslash \Lambda(x)$. As $\operatorname{Graph}\left(\Lambda^{c}\right)=\left(X \times \mathbb{R}^{d}\right) \backslash \operatorname{Graph}(\Lambda) \in \mathcal{A}\left(X \times \mathbb{R}^{d}\right)$, from Bertsekas and Shreve (2004), Proposition 7.39, page 165, we get that

$$
\left\{x \in X, \Lambda^{c}(x) \cap O \neq \varnothing\right\}=\operatorname{Proj}_{X}\left((X \times O) \cap \operatorname{Graph}\left(\Lambda^{c}\right)\right) \in \mathcal{A}(X) \subset \mathcal{B}_{c}(X) .
$$

Thus $\Lambda^{c}$ is $\mathcal{B}_{c}(X)$-measurable and as $\Lambda^{c}$ is closed valued, Rockafellar and Wets (1998), Theorem 14.8, page 648, applies and $\operatorname{Graph}\left(\Lambda^{c}\right)$ belongs to $\mathcal{B}_{c}(X) \otimes$ $\mathcal{B}\left(\mathbb{R}^{d}\right)$ and $\operatorname{Graph}(\Lambda)$ as well.

\section{REFERENCES}

Aliprantis, C. D. and Border, K. C. (2006). Infinite Dimensional Analysis: A Hitchhiker's Guide, 3rd ed. Springer, Berlin. MR2378491

Avellaneda, M., Levy, A. and Paras, A. (1996). Pricing and hedging derivatives securities in markets with uncertain volatilities. Appl. Math. Finance $273-88$.

BARTL, D. (2016). Exponential utility maximization under model uncertainty for unbounded endowments. Available at arXiv:1610.00999.

Bertsekas, D. and Shreve, S. (2004). Stochastic Optimal Control: The Discrete-Time Case. Athena Scientific, Nashua, NH. MR0809588

BLANCHARD, R. and CARASSUS, L. (2017). Quantitative fundamental theorem of asset pricing in discrete-time case with multiple priors. In preparation.

BlANChARD, R., CARASSUS, L. and RÁSONYI, M. (2016). Non-concave optimal investment and no-arbitrage: A measure theoretical approach. Available at arXiv:1602.06685. 
Bouchard, B. and Nutz, M. (2015). Arbitrage and duality in nondominated discrete-time models. Ann. Appl. Probab. 25 823-859.

CARASSUS, L. and RÁSONYI, M. (2016). Maximization of non-concave utility functions in discretetime financial market. Math. Oper. Res. 41 146-173.

CARAssus, L., RÁSONYI, M. and Rodrigues, A. M. (2015). Non-concave utility maximisation on the positive real axis in discrete time. Math. Financ. Econ. 9 325-348.

Castaing, C. and Valadier, M. (1977). Convex Analysis and Measurable Multifunctions. Lectures Notes in Mathematics 580. Springer, Berlin.

Denis, L., Hu, M. and PENG, S. (2011). Function spaces and capacity related to a sublinear expectation: Application to G-Brownian motion paths. Potential Anal. 34 139-161.

DENIS, L. and KERVAREC, M. (2013). Optimal investment under model uncertainty in nondominated models. SIAM J. Control Optim. 51 1803-1822.

DENIS, L. and MARTINI, C. (2006). A theoretical framework for the pricing of contingent claims in the presence of model uncertainty. Ann. Appl. Probab. 16 827-852.

Donoghue, W. F. (1969). Distributions and Fourier Transforms. Pure and Applied Mathematics 32. Elsevier, Amsterdam.

Föllmer, H. and Schied, A. (2002). Stochastic Finance: An Introduction in Discrete Time. de Gruyter, Berlin.

Gelbaum, B. R. and Olmsted, J. M. H. (1964). Counterexamples in Analysis. Holden-Day, Inc., San Francisco, CA. MR0169961

GilboA, I. and SchMeIDLER, D. (1989). Maxmin expected utility with non-unique prior. J. Math. Econom. 18 141-153.

JACOD, J. and ShIRYAEV, A. N. (1998). Local martingales and the fundamental asset pricing theorems in the discrete-time case. Finance Stoch. 2 259-273.

Knight, F. (1921). Risk, Uncertainty, and Profit. Hart, Schaffner Marx; Houghton Mifflin Co., Boston, MA.

KrAmkov, D. O. and SchaChERMAYER, W. (1999). The asymptotic elasticity of utility functions and optimal investment in incomplete markets. Ann. Appl. Probab. 9 904-950.

LYONS, F. (1995). Uncertain volatility and the risk-free synthesis of derivatives. Appl. Math. Finance 2 117-133.

NeUfELD, A. and SiKIC, M. (2016). Robust utility maximization in discrete time with friction. Available at arXiv:1610.09230.

NutZ, M. (2016). Utility maximisation under model uncertainty in discrete time. Math. Finance 26 252-268.

Pennanen, T. and PerkKio, A.-P. (2012). Stochastic programs without duality gaps. Math. Program. 136 91-220.

RÁsOnYi, M. and STETTNER, L. (2005). On the utility maximization problem in discrete-time financial market models. Ann. Appl. Probab. 15 1367-1395.

RÁSONYI, M. and STETTNER, L. (2006). On the existence of optimal portfolios for the utility maximization problem in discrete time financial models. In From Stochastic Calculus to Mathematical Finance (Y. Kabanov, R. Lipster and J. Stoyanov, eds.) 589-608. Springer, Berlin.

Rockafellar, R. T. (1970). Convex Analysis. Princeton Univ. Press, Princeton, NJ.

Rockafellar, R. T. and Wets, R. J.-B. (1998). Variational Analysis. Springer, Berlin. MR1491362

Sainte-Beuve, M.-F. (1974). On the extension of von Neumann-Aumann's theorem. J. Funct. Anal. 17 112-129. MR0374364

SCHACHERMAYER, W. (2001). Optimal investment in incomplete markets when wealth may become negative. Ann. Appl. Probab. 11 694-734.

SCHIED, A., Föllmer, H. and WEBER, S. (2009). Robust preferences and robust portfolio choice. In Mathematical Modelling and Numerical Methods in Finance 29-87. Elsevier, Amsterdam. 
SIERPINSKI, W. (1920). Sur un problème concernant les ensembles mesurables superficiellement. Fund. Math. 1 112-115.

von Neumann, J. and Morgenstern, O. (1947). Theory of Games and Economic Behavior. Princeton Univ. Press, Princeton, NJ.

\section{LMR}

UNIVERSITÉ REIMS CHAMPAGNE-ARDENNE

MOULIN DE LA House, BP 1039

51687 REIMS CEDEX 2

FRANCE

E-MAIL: romain.blanchard@etudiant.univ-reims.fr

\author{
RESEARCH CENTER \\ LÉONARd de Vinci Pôle Universitaire \\ 92916 PARIS LA DÉFENSE CEDEX \\ FRANCE \\ AND \\ LMR \\ UNIVERSITÉ REIMS CHAMPAGNE-ARDENNE \\ Moulin DE LA House, BP 1039 \\ 51687 REIMS CEDEX 2 \\ FRANCE \\ E-MAIL: laurence.carassus@devinci.fr
}

